\title{
(1) \\ 2iin \\ UNIVERSIDAD SANTOTOMAS \\ ANALISIS DEL SECTOR MINERO Y AGROPECUARIO Y SUS EFECTOS \\ SOBRE LOS SALARIOS Y MANO DE OBRA EN EL \\ MUNICIPIO DE SAN JOSÉ DE CÚCUTA 2002-2012
}

JUAN CARLOS MEZA MARTÍNEZ

Universidad Santo Tomas

Maestría en Ciencias Económicas

Bogotá D.C., Colombia

2014 


\section{MII \\ iin \\ UNIVERSIDAD SANTOTOMAS \\ ANALISIS DEL SECTOR MINERO Y AGROPECUARIO Y SUS EFECTOS \\ SOBRE LOS SALARIOS Y MANO DE OBRA EN EL \\ MUNICIPIO DE SAN JOSÉ DE CÚCUTA 2002-2012}

\section{JUAN CARLOS MEZA MARTINEZ}

Tesis presentada como requisito parcial para optar al título de:

Magister en Ciencias Económicas

Director:

OSCAR ARCOS PALMA

Universidad Santo Tomas

Maestría en Ciencias Económicas

Bogotá D.C., Colombia

2014 


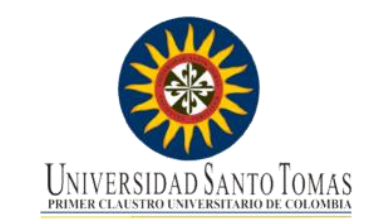

\section{Agradecimientos}

A mi Padre Carlos Arturo Meza Carvajalino por sus continuos consejos apoyo y esmero A mi madre Amira Martinez por su fe y su constancia A mis dos mis hermanas Carols y Laura A mi director Oscar Arcos y sus continuos comentarios que fue posible esta investigación 


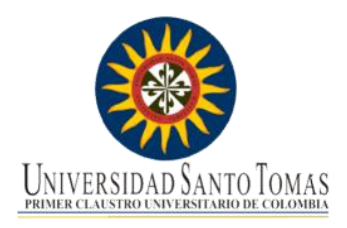

\title{
Resumen
}

Una de las actividades con mayor auge económico en el municipio de Cúcuta, es la minería, en cuanto a la explotación de carbón mineral, que produce grandes utilidades a las empresas públicas y privadas de la zona. Igualmente, la mano de obra que se emplea, en su mayoría es nativa u oriunda del municipio, lo que permite una dinámica laboral que estabiliza económicamente a la población ocupada en el sector minero. Además, presenta un crecimiento intensivo en salarios reales y de mano de obra no calificada. De otra parte, el sector agropecuario en el municipio de San José de Cúcuta, ha venido en detrimento, reduciéndose su capacidad productiva, en especial para los minifundistas, donde sus predios se encuentran entre 27 y 37 hectáreas, además de tener mano de obra no calificada y salarios reales que se encuentran son bajos en relación al sector minero. En contraste con las actividades mineras, la agricultura es poco explotada y las actividades pecuarias han perdido valor en los últimos 10 años.

Palabras clave: Minería, Agricultura, Salarios, mano de obra

\begin{abstract}
Abstrac
One of the activities with the greatest economic boom in the town of Cucuta, is mining, in terms of the exploitation of coal, which produces large profits to public and private companies in the area. Similarly, the labor force is employed, is mostly native or native of the town, allowing economically dynamic labor stabilizes the occupied population in the mining sector. It presents a real and intensive growth of unskilled labor wages. Moreover, the agricultural sector in the town of San José de Cúcuta, has been detrimental, reducing its production capacity, especially for smallholders, where their properties are between 27 and 37 hectares, in addition to unskilled labor qualified and real wages are low in relation to the mining sector. In contrast to mining, agriculture is largely untapped and livestock activities have lost value in the last 10 years.
\end{abstract}

Keywords: Mining, Agriculture, Wages, labor 
Contenido

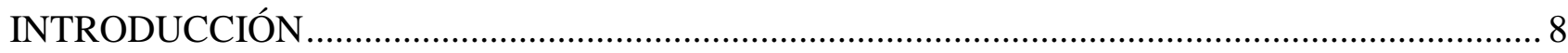

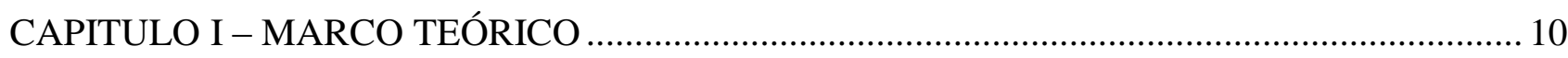

1.1. EL PARADIGMA TRADICIONAL: LA VISIÓN DE LOS EFECTOS

NEGATIVOS DE LA MINERIA EN LAS ACTIVIDADES PRIMARIAS ................. 11

1.2. EL NUEVO PARADIGMA: LA VISIÓN DE LOS EFECTOS POSITIVOS DE

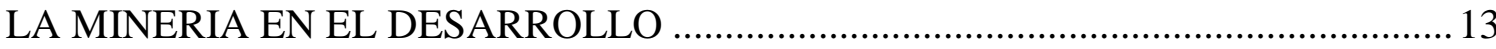

CAPITULO II - SITUACIÓN DE CONTEXTO .............................................................. 15

2.1. COEFICIENTE DE GINI PARA TIERRAS Y PROPIETARIOS DE SAN JOSÉ

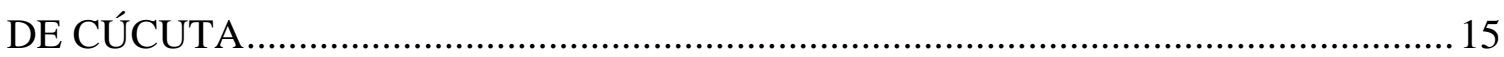

2.2.1. ACTIVIDADES MINERO ENERGÉTICAS EN SAN JOSÉ DE CÚCUTA .... 19

CAPITULO III - COMPRABACIÓN EMPÍRICA

3.1. EMPLEO DE LA ACTIVIDAD MINERA Y AGROPECUARIA.

3.1.1. RELACIÓN EMPLEO E SALARIOS EN LAS ACTIVIDADES MINERAS Y

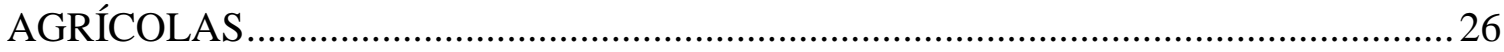

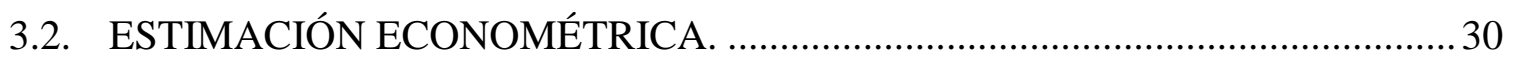

3.3. MODELO DE MÍNIMOS CUADRADOS ORDINARIOS ................................ 31

3.4. MODELO DE REGRESIONES CUANTILICAS DEL INGRESO DE LOS

SECTORES ECONÓMICOS DE SAN JOSÉ DE CÚCUTA........................................ 36

CONCLUSIONES Y RECOMENDACIONES ............................................................... 41

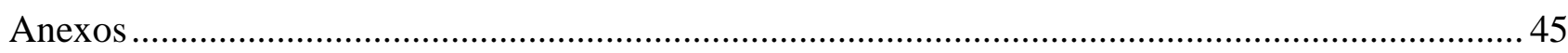

\section{INDICE DE TABLAS}

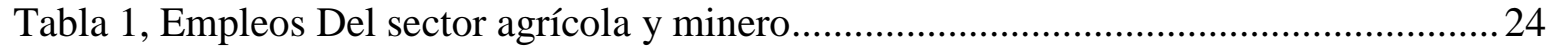

Tabla 2 Salarios reales por actividades económicas $2002-2012$....................................28

Tabla 3, Matriz de correlación con significancia estadística........................................29

Tabla 4, Regresión por Mínimos Cuadrados Ordinarios (MCO) de los salarios totales correspondientes a las ramas de actividad económica de San José de Cúcuta.....................34 
Tabla 5, Resultados de la regresión cuantilica para el logaritmo natural del salario y las

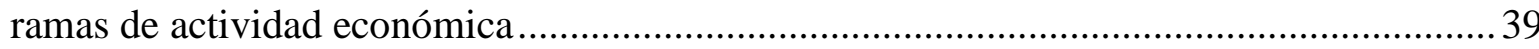

\section{INDICES DE GRÁFICOS}

Gráfico 1, tenencia de la tierra en el municipio de San José de Cúcuta 2010 .................... 17

Gráfico 2, tenencia de la tierra en el municipio de San José de Cúcuta 2010 .................... 18

Gráfico 3, Numero de hectáreas por actividad minera en el municipio de San José de

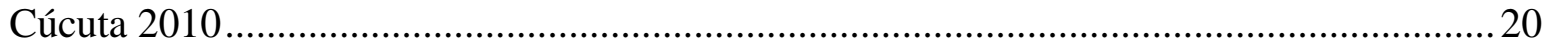

Gráfico 4, porcentaje de hectáreas correspondiente al sector minero energético de San José de Cúcuta.

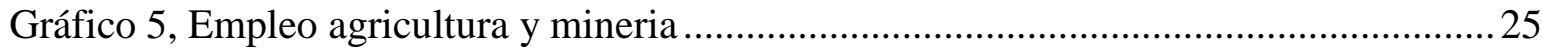

Gráfico 6, Relación entre los salarios reales per cápita y el empleo por rama de actividad económica en San José de Cúcuta .............................................................................. 26

Gráfico 7 salarios reales por rama de actividad económica en San José de Cúcuta............27

Gráfico 8, salarios per cápita del sector minero y agropecuario 2002-2012 ....................29

Gráfico 9, Estimación de los salarios reales con respecto a las actividades económicas.....35

Gráfico 10, Resultados de la regresión cuantilica para el logaritmo natural del salario y las ramas de actividad económica....

\section{INDICE DE MAPAS}




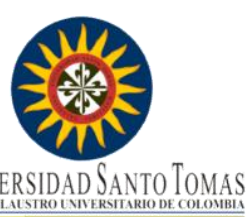




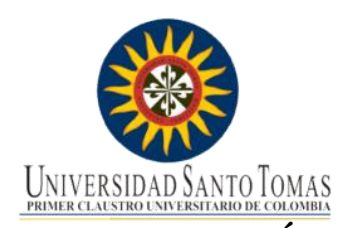

INTRODUCCIÓN

La minería ha tenido un auge notable en los últimos años a nivel mundial ${ }^{1}$. América Latina y Colombia no han sido ajenas a ese comportamiento. En Colombia, el auge se ha observado en distintas regiones. A propósito de la tendencia creciente de la participación de las inversiones en el sector minero y de su mayor participación en el producto interno bruto, se ha centrado la atención en la discusión teórica acerca de si la actividad minera produce efectos positivos o efectos negativos en actividades económicas sectoriales que comparten territorios. En términos amplios se ha dicho recientemente que la minería puede ser motor de desarrollo y que su dinamismo puede generar impactos económicos y sociales favorables en otros sectores de la economía. La hipótesis que se ha planteado para esta investigación es que la actividad minera, al contrario de lo que se afirma, produce efectos negativos en sectores que comparten territorio, en particular en el sector agrario.

Este trabajo de investigación se ha propuesto elucidar, en un estudio de caso, la realidad de esas afirmaciones, en una región particular donde se ha observado en la última década un crecimiento importante de la actividad minera: San José de Cúcuta. En razón a que parte del territorio que delimita a San José de Cúcuta se destina a las actividades mineras y parte a las actividades agrarias, la investigación se ha encaminado a establecer los posibles efectos que el auge minero en San José de Cúcuta haya producido sobre la actividad agraria. Para el efecto, la investigación propone un análisis de las variables empleo e ingresos en los dos sectores.

\footnotetext{
${ }^{1}$ Fundación para la educación superior y el desarrollo -FEDESARROLLO- Cárdenas Mauricio, Reina Mauricio, Rubiano Eliana, Rozo Sandra y Becerra Oscar (2008), La minería en Colombia: impacto socioeconómico y fiscal. Proyecto de la Cámara ASOMINEROS de la ANDI. El estudio de Fedesarrollo señala que desde 2002 se ha registrado un auge global de las materias primas, que ha significado que los precios internacionales de varios metales hayan crecido más del doble en ese lapso. Destaca que dicho auge ha durado más que el promedio de las bonanzas de commodities que se han registrado en los últimos 30 años, y que además es probable que se mantenga algunos años más gracias a la expansión sostenida de China y a las perspectivas de crecimiento de la India.
} 
De acuerdo con el párrafo anterior, las actividades económicas que se ejercen en los terrenos de Cúcuta, se pueden medir a través de los precios hedónicos que en algunas propiedades donde se generan la producción minera son claramente desproporcionados, con relación a aquellos predios con actividades agropecuarias. Por tanto, surge la siguiente pregunta de investigación iha sido el auge del sector minero el que ha reducido la capacidad productiva y el crecimiento de las actividades agropecuarias en San José de Cúcuta en el periodo de 2002-2012?

En el primer capítulo se exponen los argumentos teóricos que dan sustento a la investigación; el segundo contiene información de contexto sobre la actividad minera y la actividad agraria en San José de Cúcuta; el tercer capítulo registra los ejercicios empíricos, por medio de la estadística descriptiva y modelos de estimación econométrica conocidos como los Mínimos Cuadrados Ordinarios MCO y de regresión cuantilica. Finalmente se exponen las conclusiones. 


\section{VIV \\ $\frac{1}{4}$ \\ Universidad SANTOTOMas

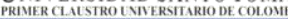 \\ CAPITULO I - MARCO TEÓRICO}

Son múltiples las interrelaciones entre sectores económicos y también lo son las relaciones entre dinámicas económicas de orden nacional, regional o local. De esas interrelaciones dependen la formación de los precios y el comportamiento de la oferta y la demanda de bienes y servicios. En particular, las dinámicas productivas y las tendencias sectoriales explican en cierta forma el comportamiento del empleo y los salarios. A propósito de ésta última afirmación, en la teoría económica existe el argumento de que las fluctuaciones del producto determinan los cambios en el empleo. En Colombia, esta apreciación ha sustentado estudios diversos y uno de los pioneros fue el de la Misión del Empleo, realizado en 1986, en el que se podía observar el supuesto teórico mencionado.

El soporte teórico para observar interrelaciones entre dos o más sectores y los efectos que pueden ocasionar en el empleo y el ingreso, se encuentra en una visión funcionalista de la economía $^{2}$. En la literatura económica se ha dicho que las teorías tradicionales del desarrollo económico suelen ofrecer una perspectiva crítica de la actividad minera ${ }^{3}$. De acuerdo a esas posturas teóricas, la actividad de la minería no hace aportes significativos al proceso de desarrollo de un país o de una región; al contrario, se dice que dicha actividad en cierta forma caracterizada por el uso de tecnologías de punta-, deriva en efectos negativos en el comportamiento de otros sectores económicos. Esta apreciación sobre el rol negativo de la minería en otros sectores económicos se aplica de manera particular a las actividades primarias, incluida la agricultura.

De acuerdo con Rubiano et al (2008), la minería es uno de los sectores que en países como Estados Unidos permite un adecuado desarrollo que coadyuva a un elevado crecimiento económico. Sin embargo, en países con economías emergentes, donde se da la presencia de actividades formales e informales, en las primeras se puede observar ciertos efectos positivos en materia de salarios, pero no de número de empleos, mientras en las actividades

\footnotetext{
${ }^{2}$ Entre otros autores, Bruce, Johnston y Mellor (1961)

${ }^{3}$ Power Thomas Michael, (2002), Digging to Development? A Historical Look at Mining and Economic Development. Oxfam Amercian Report. Véase también Rubiano et al (2008)
} 
mineras informales se dan condiciones que perpetúan la pobreza. Otros autores (v. gr., Hajkowicz et al., 2011), señalan que el impacto positivo que ocasionan las actividades mineras es "relativo" en materia de empleo y de ingresos dentro del mismo sector y también con relación a los efectos que puede ocasionar en otros sectores económicos. La relación de impactos positivos del sector minero quizás pueda observarse con mayor claridad con respecto al sector manufacturero, y no tanto con respecto al sector agrario.

En otra perspectiva, se sabe que mientras que las actividades mineras coadyuvan al crecimiento económico del país, las actividades agropecuarias presentan ciclos económicos y su aporte a la economía es inferior al que hacen las actividades mineras. De acuerdo con la Gran Encuesta Integrada de Hogares del DANE, los números de empleos que se crean en el sector minero y agropecuario son bajos en relación con las actividades del tercer sector como el comercio y los servicios. Sin embargo, los ingresos per cápita del sector minero son mayores en relación al sector agropecuario, principalmente por la tecnología desarrollada.

En general las tecnologías más desarrolladas y en especial aquellas que optimizan los recursos y maximizan los beneficios en el sector minero, está a merced de la naturaleza donde no existen sustitutos y de esta manera es totalmente diferente a las demás industrias. Por tanto, es posible inferir que el auge del sector minero deteriora otras actividades cuya producción es tomada directamente de la tierra como la agricultura, y asimismo los ingresos por cápita de la misma.

\subsection{EL PARADIGMA TRADICIONAL: LA VISIÓN DE LOS EFECTOS NEGATIVOS DE LA MINERIA EN LAS ACTIVIDADES PRIMARIAS}

Son diversos los autores que discuten acerca de la apreciación negativa del papel de la minería en sectores como la agricultura ${ }^{4}$. En primera instancia se presentan los argumentos

\footnotetext{
${ }^{4}$ Entro otros, Singer (1950), Prebisch (1959), Mauro (1995), Tornell y Lane (1999), Collier y Hoffler (2002), Davis (2002), Sala-i-Martin (2003), Banco Mundial (2007), Banco Mundial (2006), Banco Mundial e IFC (2002), Alexeev y Conrad (2005), Auty (2000), Stijins(2001), Power et al. (2002
} 
de quienes señalan que la minería no sólo no genera efectos importantes en el desarrollo, sino que perjudica a otros sectores, especialmente a los de actividades primarias:

- La primera vertiente conceptual es la que advierte sobre la tendencia descendente de los productos básicos en el mercado internacional. Los autores de esta vertiente afirman que, más allá de las oscilaciones naturales de corto y mediano plazo, en el largo plazo los precios internacionales de los productos básicos registran una tendencia secular hacia el descenso. La principal causa económica de esta tendencia consiste en que los productos primarios tienen una baja elasticidad ingreso, sobre todo comparada con la de los productos manufacturados. De esta manera, a medida que los países aumentan sus niveles de ingreso, tienden a orientar una mayor porción relativa de su demanda hacia manufacturas, en detrimento de los productos primarios como los alimentos y las materias primas. Estas apreciaciones se han observado en Colombia, incluso desde la dinámica de transformación del mercado interno desde los años cincuenta del siglo XX, cuando se demostró que los productos agrarios tienen una baja elasticidad con respecto a la demanda. A medida que los hogares disponen de mayores ingresos, las proporciones de los ingresos destinados al consumo de bienes agrarios de consumo desciende.

- La segunda vertiente conceptual se concentra en los efectos macroeconómicos perversos que puede tener el auge de un sector primario sobre el resto del aparato productivo, mejor conocidos en los debates económicos como la enfermedad holandesa. En principio puede haber dos mecanismos a través de los cuales el auge de un sector primario podría generar efectos nocivos sobre el resto de la economía. El primero está asociado con la tasa de cambio. En la medida en que un país tenga una bonanza externa de un producto primario, contará con una súbita abundancia de divisas, lo que reducirá la tasa de cambio y hará que el resto de la economía pierda competitividad en los mercados internacionales y en el nacional frente a los productos importados. El segundo mecanismo a través del cual el auge de un sector primario puede afectar negativamente al resto de la economía tiene que ver con la asignación de los recursos productivos. En la medida en que el sector primario en auge se vuelve más rentable, extrae recursos 
productivos (en especial trabajo) del resto de actividades, afectando negativamente su productividad y su desempeño.

- La tercera vertiente analítica que sugiere que las actividades primarias (y en concreto la minería) no tendrían un efecto favorable sobre el desarrollo económico se concentra en el análisis de diversas condiciones económicas y políticas del país en cuestión. Por un lado, estos planteamientos sugieren que la minería no generaría mayor impacto sobre el resto de la economía de un país cuando no tienen muchos eslabonamientos con el resto de la economía hacia atrás ni hacia adelante.

\subsection{EL NUEVO PARADIGMA: LA VISIÓN DE LOS EFECTOS POSITIVOS DE LA MINERIA EN EL DESARROLLO}

El paradigma tradicional sobre el papel de la minería en el desarrollo, que advierte que el sector tiene un impacto nulo o negativo sobre el crecimiento del resto de la economía, ha sido cuestionado en años recientes. La revisión crítica de este enfoque ha sido el resultado de la observación que se ha hecho durante las últimas décadas en países desarrollados donde el sólido desarrollo de la minería ha ido de la mano de importantes niveles de crecimiento económico. Desde luego, el paradigma se sustenta en apreciaciones que remiten a la calidad de las instituciones de esos países, al manejo razonable de la política macroeconómica y las políticas gubernamentales relacionadas con la educación, la formación del capital humano y las inversiones en ciencia y tecnología. Este paradigma se sustenta en el hecho de que durante las últimas décadas las compañías mineras internacionales han multiplicado sus inversiones en exploración y explotación alrededor del mundo.

El informe Proyect Survey ${ }^{5}$ del año 2002, señala que el gasto global en la exploración de minerales no ferrosos ha aumentado de 1.900 millones de dólares en 2002 a 5.000 millones de dólares en 2005. Lo que hace más relevante este auge internacional de la minería es que

\footnotetext{
5 'Project Survey 2002', en Engineering and Mining Journal 203, Enero 2002
} 
América Latina ha tenido un papel preponderante en medio del dinamismo de los procesos de exploración, hasta el punto que de acuerdo con algunos análisis figura como la región minera de mayor crecimiento en el mundo. Este es el argumento que permite inferir la existencia de un nuevo paradigma, bajo el entendido que las inversiones y las innovaciones tecnológicas, en un contexto de políticas adecuadas y manejo apropiado de las variables macroeconómicas, colocan al sector minero como un impulsor del desarrollo económico y social de un país.

Con respecto a la segunda vertiente mencionada en el paradigma tradicional, los argumentos del nuevo paradigma señalan que los efectos macroeconómicos nocivos que puede tener la bonanza de un sector primario se contrarrestan con propuestas de políticas para neutralizarlos, entre ellas las direccionadas a neutralizar los efectos cambiarios de una bonanza exportadora. Al respecto, se suele afirmar que la intervención de la autoridad monetaria en el mercado cambiario para evitar que caiga el precio de la divisa, esa opción no es sostenible en el mediano plazo por los efectos inflacionarios que esa intervención genera. En este sentido, la alternativa más idónea es la creación de un fondo de retención de divisas en el exterior, que permita regular su ingreso a la economía y neutralizar su efecto sobre la tasa de cambio. La segunda propuesta de política que se suele formular en estos casos busca dar apoyo a los sectores de la economía que se pueden ver perjudicados ante la bonanza de un producto específico, ya sea mediante créditos especiales con tasas bajas y plazos largos, o a través de mecanismos de cobertura o compensación cambiaria.

Los argumentos que se suelen plantear para hacer frente a los problemas de la tercera vertiente analítica presentada en la sección anterior son de variada índole. Por un lado, como se verá más adelante, la minería puede abarcar actividades con diverso grado de agregación de valor, desde la mera extracción del mineral hasta procesos productivos complejos y elaborados. En este sentido, las políticas a aplicar deberían fomentar actividades con alto grado de agregación de valor en lugar de las simples labores extractivas. De otro lado, es posible potenciar los encadenamientos de la actividad minera 
mediante el desarrollo de clusters de actividades afines, algunas de las cuales pueden ser alta tecnología como el diseño y la fabricación de maquinaria especializada.

Al analizar la dimensión sectorial se encuentra que estas economías han desarrollado una minería con grandes eslabonamientos hacia otras actividades productivas, que han permitido jalonar mayor empleo y valor agregado, y que han promovido el surgimiento de clusters productivos alrededor de polos de desarrollo minero. Finalmente, en todos los casos exitosos se han promovido políticas específicas de formación de capital humano que han permitido elevar la productividad y la capacidad tecnológica de las actividades productivas de la minería y de los clusters circundantes.

\section{CAPITULO II - SITUACIÓN DE CONTEXTO}

En este capítulo se presentará las características generales de la actividad minera y agropecuaria en el municipio de San José de Cúcuta en cuanto a su relación con la estructura de la propiedad y el grado de concentración de la tierra explicada por medio del coeficiente de GINI.

En este sentido, se tomó como variable proxi la concentración de la tierra en San José de Cúcuta y su actividad en dos momentos, en el periodo 2002- 2011, tomados de los datos del

Instituto Geográfico Agustín Codazzi, el Plan de Ordenamiento Territorial- POT etc., en donde se tomó información relevante para el siguiente análisis.

\subsection{COEFICIENTE DE GINI PARA TIERRAS Y PROPIETARIOS DE SAN JOSÉ DE CÚCUTA}

En ese sentido, San José de Cúcuta, posee un alto grado de concentración de la tierra, dado que se registra como una de las ciudades con mayor inequidad y mayor concentración en la distribución de la tierras GINI, el cual se ubica en el rango de 0,73 y 0,89 en el año 2010 según el estudio realizado por el convenio de la pontificia Universidad Javeriana "Atlas de la Distribución de la Propiedad Rural en Colombia” 
De acuerdo con lo anterior, el índice de GINI de tierras presenta una alta concentración de las mismas. Asimismo, el DANE reporta el índice de GINI desde al año 2008 y 2009 con 0,473 y 0,525 respectivamente para propietarios, y para el año 2010 se encuentra entre el rango 0,63 y 0,73, según el informe del IGAC (2011), lo que se evidencia una concentración de tierras en manos de propietarios. Aunque, este último demuestra que la mayoría de las tierras están en manos en pocos propietarios.

Por otro lado, para caracterizar la estructura de la propiedad, se toma como unidad de medida la unidad agrícola familiar -UAF, definida por el Instituto Colombiano de Desarrollo Rural -INCODER, y es aquella que básicamente emplea mano de obra del propietario o poseedor y su familia inmediata constituido por esposa e hijos. Ésta, emplea economías de autoconsumo o de subsistencia y cuya producción es suficiente para suplir las necesidades básicas de la familia.

Las UAF, institucionalmente están definidas en la Ley 60 de 1994 y en la Resolución 41 de 1996 del Instituto Colombiano de Reforma Agraria -INCORA, la cual define los tamaños de las UAF, de acuerdo a la caracterización de las zonas y uso del suelo en cuanto a actividades como la agricultura, ganadería, pesca entre otros.

Entre mayor sean los tamaños de los predios, es posible evidenciar actividades económicas como la agroindustria, la minería, la extracción de combustibles fósiles entre otros, toda vez que los cambios y avances tecnológicos permitan la producción a gran escala. Por consiguiente, para conocer como está definida la estructura de la propiedad, se toma como unidad de medida la unidad agrícola familiar-UAF-, que institucionalmente están definidas en la Ley 60 de 1994 y en la Resolución 41 de 1996 del INCORA, la cual define los tamaños de las UAF's, de acuerdo con la caracterización de las zonas y uso del suelo.

De acuerdo con el INCODER, una UAF en el municipio de San José de Cúcuta se clasifica en el rango de 33 a 44 hectáreas. Por tanto, para definir y afirmar la existencia de 
minifundio, pequeña propiedad y latifundio, la categorización de la estructura de la propiedad se delimita de la siguiente manera:

Microfundio: Son las propiedades menores a una UAF, es decir, terrenos con extensiones hasta de 32 has.

Minifundio: Aquellas propiedades igual a una UAF, entre 33 a 44 hectáreas.

Mediana Propiedad: Comprende los predios entre dos a cuatro UAF, entre 45 a 176 hectáreas.

Latifundio o Gran Propiedad: Comprende los predios de más de cuatro UAF, mas de 177 hectáreas.

Así, el propósito fundamental de este aparte es presentar los análisis de la estructura de la propiedad de la tierra rural en el municipio de San José de Cúcuta, en donde el área geográfica total es de $1.176 \mathrm{~km} 2$, donde para el años 2002, según el diagnóstico rural presentado por la alcaldía de municipal de Cúcuta, existían predominios de microfundios y minifundios que constituían el $63 \%$ del total de predios, y los predios de menor extensión pertenecían a un porcentaje menor de propietarios el cual es presentado en el Gráfico 2.

Gráfico 1, tenencia de la tierra en el municipio de San José de Cúcuta 2010

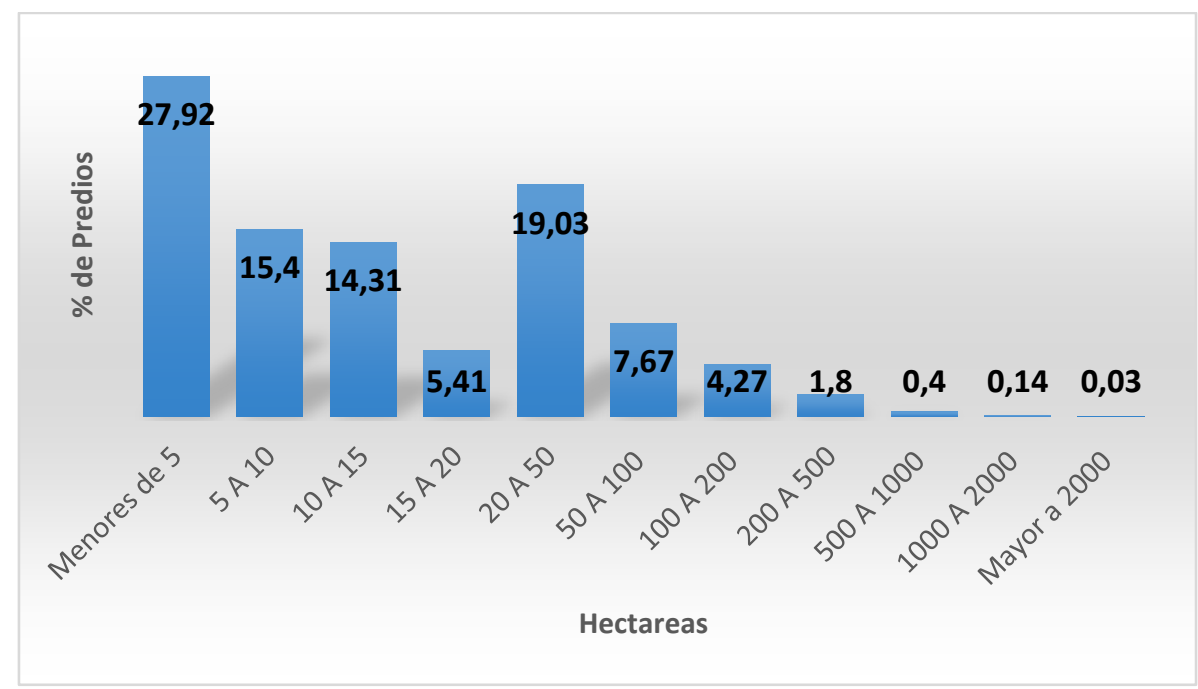

Fuente: Plan de Ordenamiento Territorial, tomado de información Alcaldía de San José de Cúcuta Departamento Administrativo Área de Planeación Corporativa y de Ciudad (2010) 
Las actividades, en pequeñas propiedades son destinadas a cultivos de pan coger y de economía de subsistencia, según lo describe Romero (2008) en el informe "lineamientos para una política de seguridad alimentaria y desarrollo regio local para la ciudad de Cúcuta". De otro lado, y en el mismo informe las grandes propiedades rurales $(2.37 \%)$ son utilizadas para la agroindustria donde los principalmente al cultivo de arroz y a la ganadería.

Para el año 2010, el informe del IGAC presenta que la concentración de la tierra se centre en los medianos y grandes propietarios, con 55\% y 35\%, respectivamente. Como se observa en el Gráfico 2.

\section{Gráfico 2, tenencia de la tierra en el municipio de San José de Cúcuta 2010}

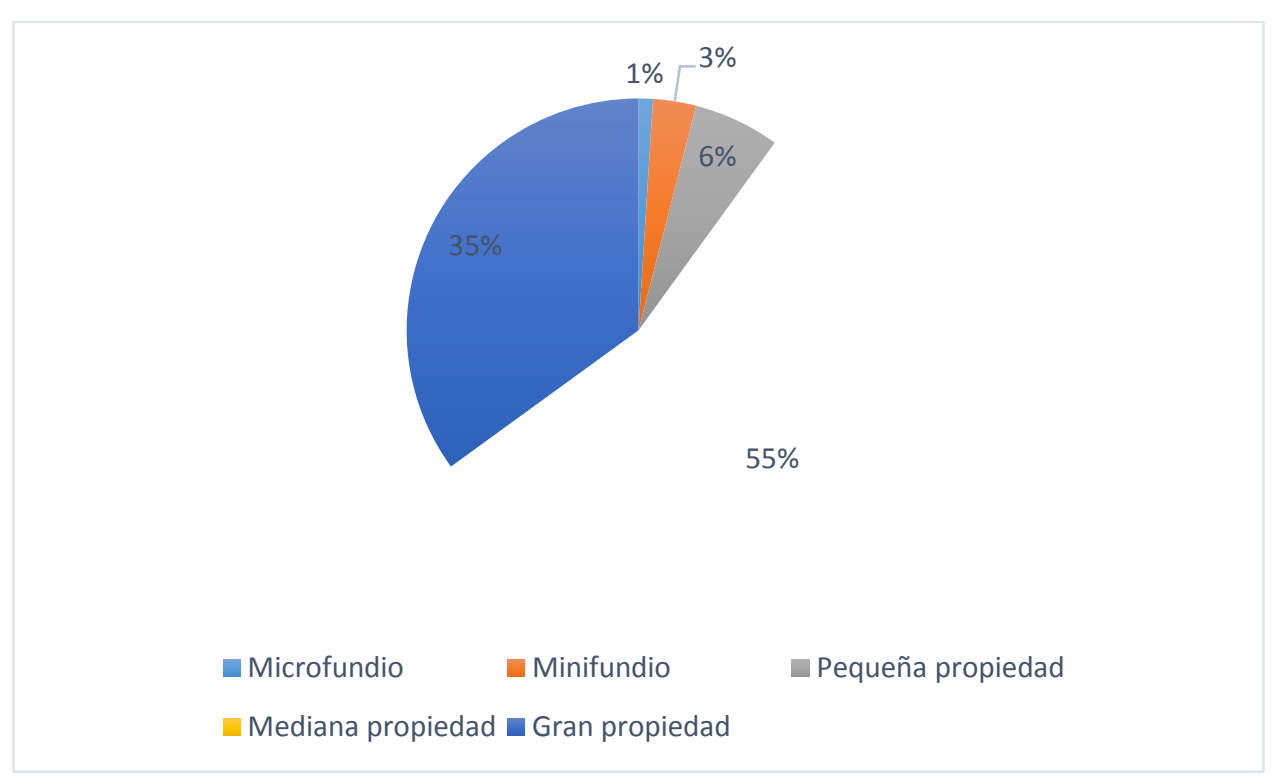

Fuente: Agustín Codazzi, Atlas de la Distribución de la Propiedad Rural en Colombia (2010)

De otra parte, las medianas y grandes propiedades (latifundios), representan el $90 \%$ del total de hectáreas existentes en el municipio de San José de Cúcuta. Los demás predios como microfundios y pequeñas propiedades suman el $7 \%$ del total de los predios en San José de Cúcuta. 
Así de esta manera, se evidencia tanto gráficamente como a través del índice de GINI que en San José de Cúcuta se concentra la tierra en pocos propietarios. Asimismo, estas tierras están dirigidas a las actividades minero energéticas como se analizará a continuación. Como se observó anteriormente la concentración de la tierra por medio del índice de GINI, ha venido aumentando, solo analizando el periodo de 2008-2010, de modo que se comprueba que la distribución de la tierra cada vez es menor.

\subsubsection{ACTIVIDADES MINERO ENERGÉTICAS EN SAN JOSÉ DE CÚCUTA}

Debido a que las grandes propiedades se centran las actividades minera, extracción de petróleo (propias del segundo sector de la economía), estas ocupan la mayoría de hectáreas en el municipio de San José de Cúcuta. Tal y como lo representa el Mapa 1.

Mapa 1, Actividades minero energéticas en el municipio de San José de Cúcuta

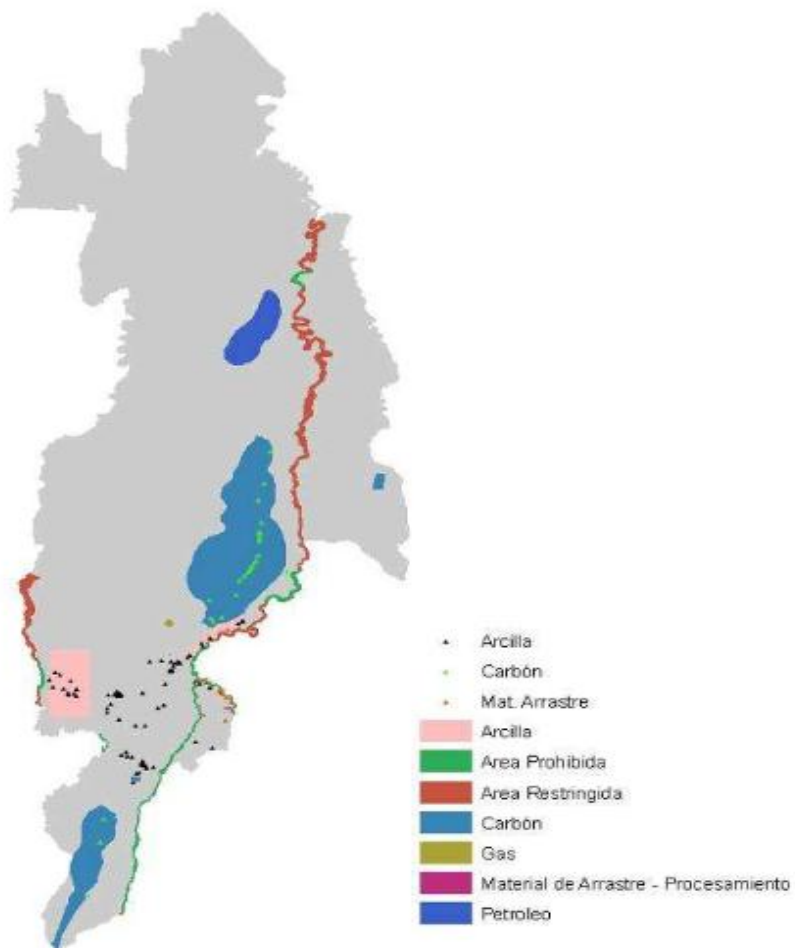

Fuente: Plan de Ordenamiento Territorial, tomado de información Alcaldía de San José de Cúcuta Departamento Administrativo Área de Planeación Corporativa y de Ciudad (2010) 
En este sentido, se puede observar en el Gráfico 3, que existen 8.166 hectáreas destinadas para la extracción de carbón, en donde se concentran en medianas y grandes propiedades (latifundios). Asimismo, las hectáreas totales para el sector minero energético suman 11.398 has.

\section{Gráfico 3, Numero de hectáreas por actividad minera en el municipio de San José de Cúcuta 2010}

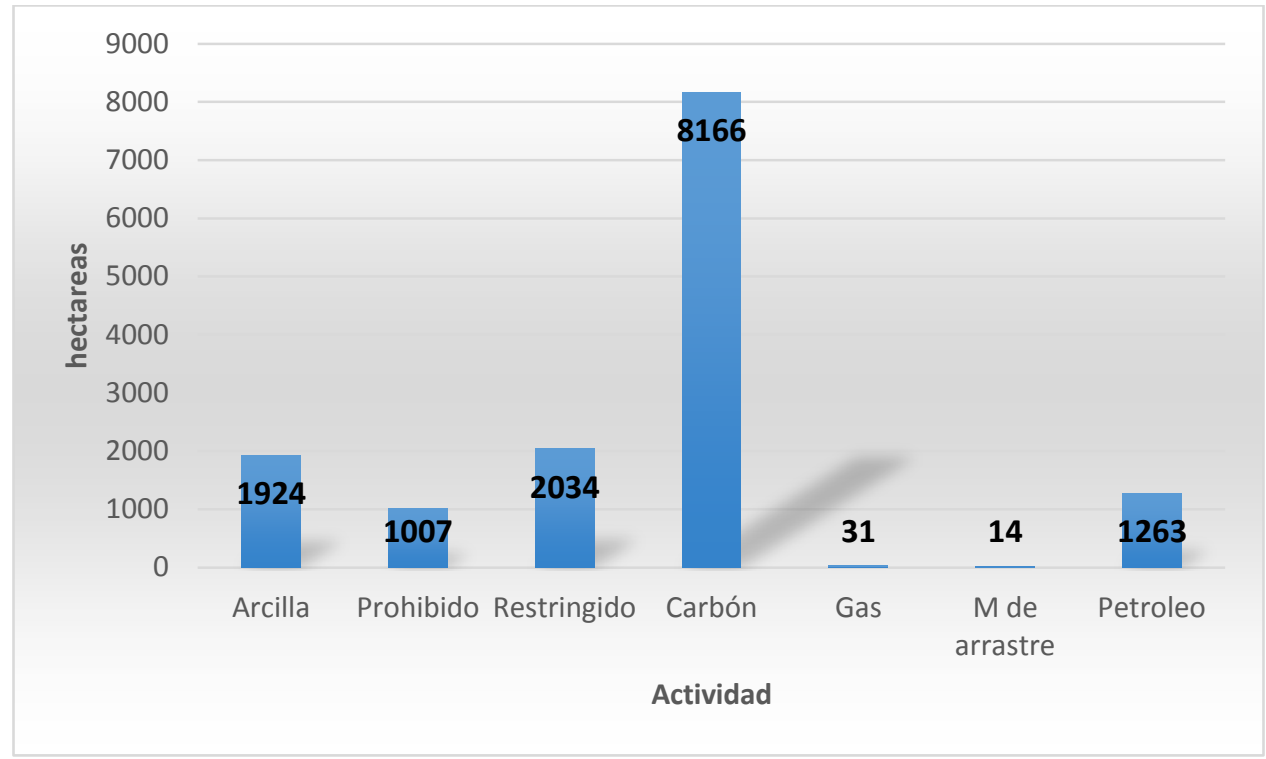

Fuente: Plan de Ordenamiento Territorial, tomado de información Alcaldía de San José de Cúcuta Departamento Administrativo Área de Planeación Corporativa y de Ciudad (2010), diseño propio

El porcentaje de hectáreas destinadas para el carbón es de 56,5\%, de 14.439 has destinadas a la explotación de minas, siendo el porcentaje más alto del segundo sector de la economía, en el caso puntual del minero energético. Asimismo, la explotación de arcilla comprende el 13\%, la extracción de petróleo se encuentra en el 8,7\%, como lo presenta el Gráfico 4. 


\section{UNIVERSIDAD SANTOTOMAS}

Gráfico 4, porcentaje de hectáreas correspondiente al sector minero energético de San José de Cúcuta.

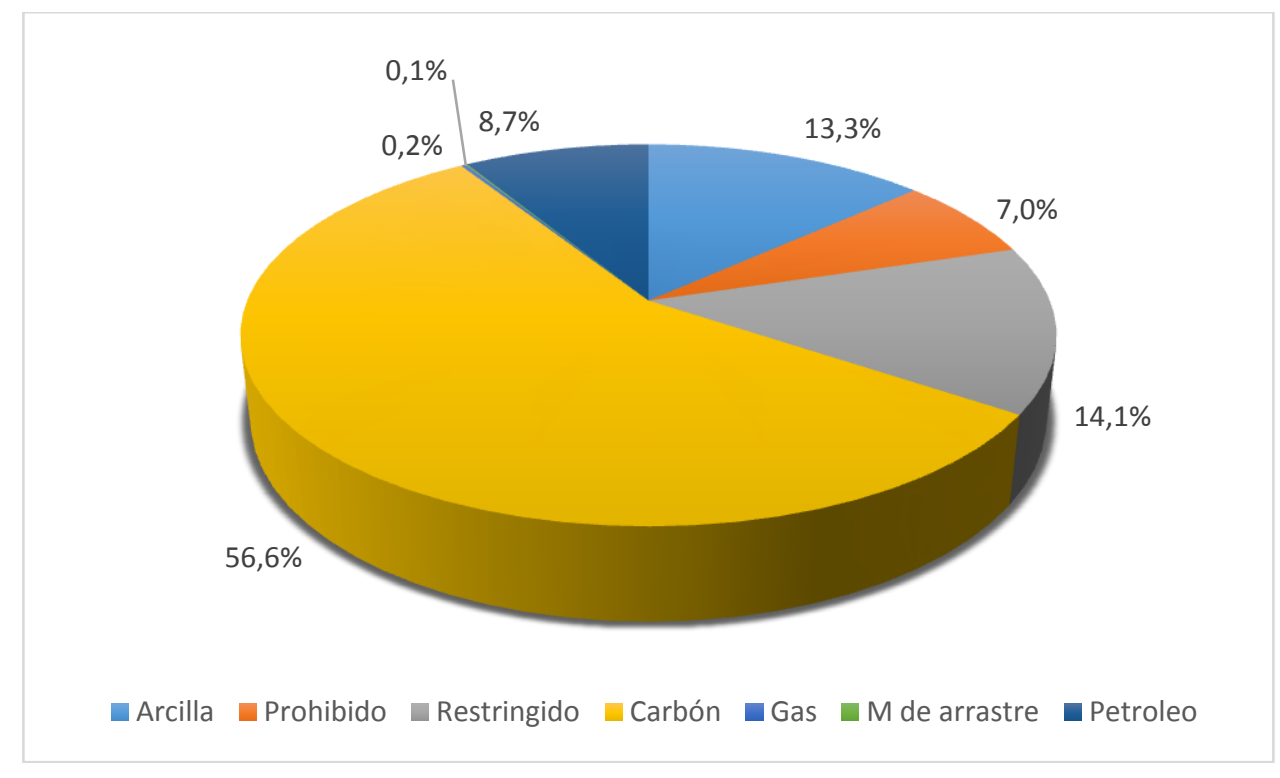

Fuente: Plan de Ordenamiento Territorial, tomado de información Alcaldía de San José de Cúcuta Departamento Administrativo Área de Planeación Corporativa y de Ciudad (2010), diseño propio

En este ítem, se analizará el sector agropecuario en el municipio de San José de Cúcuta y su relación con el crecimiento económica de la zona donde se tomarán como referencia los datos de las cuentas departamentales del DANE (2002-2013), el Plan Municipal de Desarrollo - PDM (2008-2011), y estudios realizados para las actividades del primer sector de la economía ${ }^{6}$ en el municipio de San José de Cúcuta.

En conformidad a lo anterior, el PDM de San José de Cúcuta (2008-2011) en su página 162, arguye que las economías del primer sector de la economía de la zona, presenta una baja rentabilidad agropecuaria, produciendo de esta manera que las economías de

\footnotetext{
${ }^{6}$ Según la definición del Banco de la Republica, el primer sector de la economía son todas las actividades económicas tomadas de la tierra sin ningún proceso de transformación. Dentro de este sector se encuentran la agricultura, la ganadería, la silvicultura, la caza y la pesca. Cabe aclarar que no se incorporan la minería y a la extracción de petróleo, las cuales se consideran parte del segundo sector. (Biblioteca Virtual Luis Ángel Arango). APA public. Revisado el 19 de septiembre de 2013 Web.

http://www.banrepcultural.org/blaavirtual/ayudadetareas/economia/econo53.htm
} 
subsistencia o campesinas se vean obligadas a abandonar sus actividades y desparecer su franja agrícola. En consecuencia, se han generado conflictos, situaciones de vulnerabilidad y altos costos de producción, con una oferta desigual de servicios sociales y equipamientos y altos costos familiares de transporte.

De otra parte, Según el PDM de San José de Cúcuta (2008-2011) se presenta una restricción sobre el desarrollo productivo rural, debido a la estructura educativa, como extensión del modelo urbano; además de la baja competitividad social y del acceso al empleo. Dado que existen desventajas educativas de la población rural con respecto a la urbana, los intercambios poblacionales entre las dos zonas vulneran el capital humano y las economías de producción doméstica.

Un fenómeno que surge de lo descrito en el párrafo anterior es la sustracción de la población rural joven por falta de alternativas en sus propios corregimientos, aumentando la demando de mano de obra joven y una disminución en la oferta, que conlleva a una reducción de los factores de producción.

Para el caso de la minería, en donde el crecimiento económico lo determina la transformación de los factores, como el trabajo y el capital, el municipio de San José de Cúcuta está conformado por baja mano de obra e intensivo en capital físico. Por tanto, el sector minero se constituye por ser intensiva en capital y en las tecnologías asociadas como la mano de obra calificada el cual coadyuva al crecimiento de la producción.

De acuerdo con lo anterior, el éxito de la actividad minera en San José de Cúcuta, se debe principalmente, porque los costos de producción en cuanto a la incorporación de la mano de obra es muy baja. Generalmente, se emplea mano de obra tecnificada lo que permite un aumento en el valor agregado que se le proporciona a la minería, generando aumentos en el producto.

Por el lado, en las actividades agropecuarias se utiliza poca fuerza laboral de acuerdo con datos DANE (2012) (generalmente no tecnificada), el capital físico no es intensivo y la 
tecnología es rudimentaria. Asimismo, el informe de coyuntura económica regional-ICER (2011), menciona que de los sectores con más fuente de riqueza del municipio de Cúcuta, es el primario con la agricultura, con productos como el algodón, el arroz, el tabaco, el cacao, la caña de azúcar, el café y el trigo. En este sentido, según datos DANE la agricultura, silvicultura, caza y pesca representó el 2,2\%. Sin embargo, es el sector que en proporción al tamaño de su producción, fue el que menos creció en el periodo 2010-2011.

\section{CAPITULO III - COMPRABACIÓN EMPÍRICA}

En el capítulo del Marco Teórico se hizo referencia a los dos paradigmas teóricos que explican efectos positivos o negativos de la minería sobre el comportamiento de otros sectores económicos. De esas posturas teóricas, la que se acoge para el análisis del caso de San José de Cúcuta, es la del paradigma de la visión tradicional, en particular la referida a la segunda vertiente y más específicamente, dentro de esta segunda vertiente, al segundo mecanismo, que señala que el auge de un sector primario (en este caso el de la minería) puede afectar negativamente al resto de la economía. En la medida en que el sector primario en auge se vuelve más rentable, extrae recursos productivos (en especial trabajo) del resto de actividades, afectando negativamente su productividad y su desempeño.

\subsection{EMPLEO DE LA ACTIVIDAD MINERA Y AGROPECUARIA}

De acuerdo con los datos DANE, a partir del periodo anual de 2001-2012, el empleo en el sector agrícola tuvo su mayor crecimiento a partir del periodo de 2002-2004, crandose hasta 1150 empleos en el año 2003, como lo presenta la Tabla 1, en donde se observa claramente el número de empleos creados. 


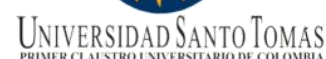

Tabla 1, Empleos Del sector agrícola y minero

\begin{tabular}{|r|r|r|}
\hline \multicolumn{1}{|c|}{ Año } & \multicolumn{1}{|c|}{$\begin{array}{c}\text { Empleos } \\
\text { Agricultura }\end{array}$} & $\begin{array}{c}\text { Empleos } \\
\text { minería }\end{array}$ \\
\hline 2001 & 619 & 149 \\
\hline 2002 & 1070 & 433 \\
\hline 2003 & 1150 & 376 \\
\hline 2004 & 1000 & 465 \\
\hline 2005 & 966 & 607 \\
\hline 2006 & 827 & 499 \\
\hline 2007 & 589 & 307 \\
\hline 2008 & 541 & 312 \\
\hline 2009 & 881 & 301 \\
\hline 2010 & 805 & 325 \\
\hline 2011 & 825 & 240 \\
\hline 2012 & 730 & 298 \\
\hline
\end{tabular}

\section{Fuente: DANE, GEIH 2001-2002 diseño propio}

Por otro lado, la minería que tuvo el mayor nñumero de empleos creados en el peri0do 2004-2006, siendo el año 2005 el periodo donde las actividades mineras tuvieron su mayor crecimiento con 607 empleos creados. Asimismo, el

Gráfico 5 presenta el comportamiento de los empleos creados en las actividades económicas en mención en San José de Cúcuta. 
Gráfico 5, Empleo agricultura y minería

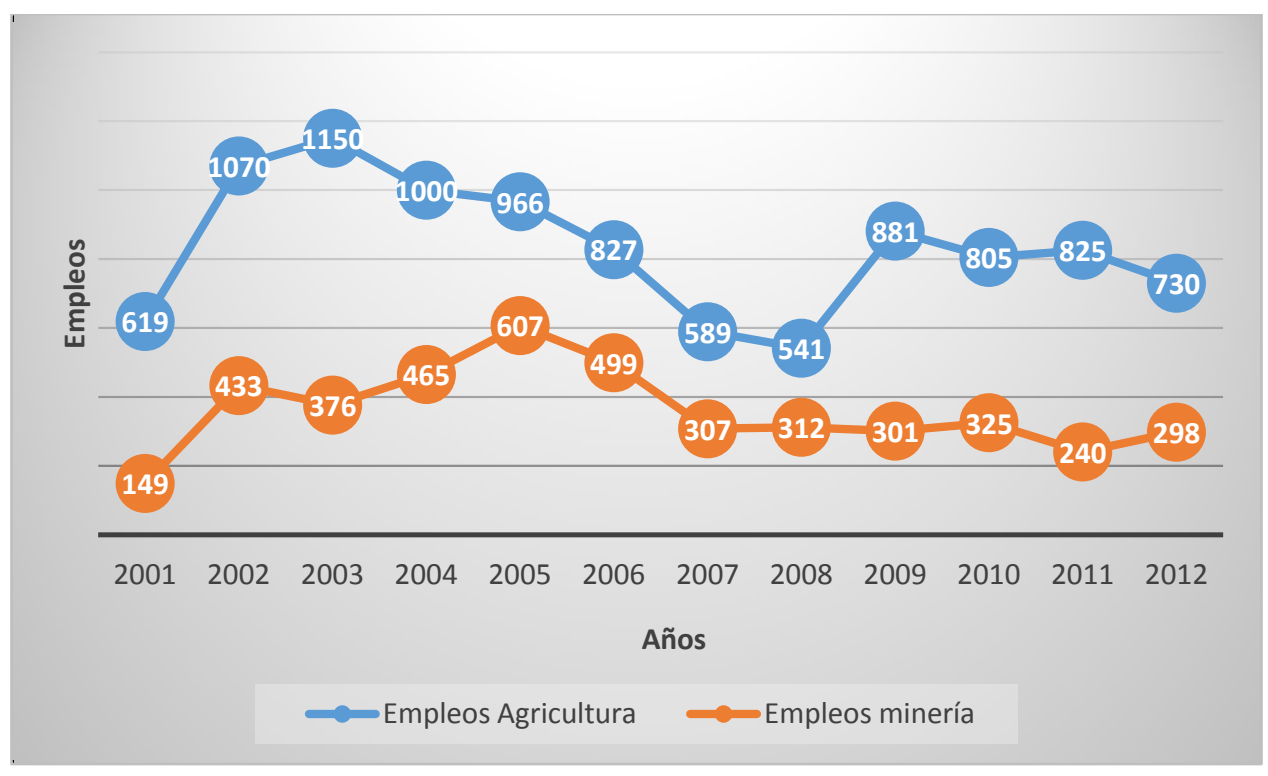

Fuente: DANE, GEIH 2001-2002 diseño propio

Como se observa en el 
Gráfico 5, las actividades agropecuarias, presento una disminución importante de empleos a partir del año de 2003 hasta el año 2008, mientras que la ocupación en la minería se ha mantenido moderadamente estable a partir del año 2007, a diferencia del sector agrícola, que tuvo un aumento de 881 empleos los cuales volvieron a descender hasta el año 2012.

De acuerdo con lo anterior, puede evidenciarse (de acuerdo con los datos DANE de la GEIH) que las actividades económicas correspondientes a la agricultura, presenta mayores ciclos de crecimiento y decrecimiento de empleos, donde se observa que la disminución de la ocupación es mayor que la creación de trabajo en este sector.

Por otro lado, en el sector minero, se presenta periodos cortos de disminución de mano de obra, como se observa en el año 2005. A diferencia del sector agrario, la minería (de acuerdo con el DANE) existe una mayor estabilidad en la inclusión de mano de obra.

\subsubsection{RELACIÓN EMPLEO E SALARIOS EN LAS ACTIVIDADES MINERAS Y AGRÍCOLAS}

Aunque la agricultura y la minería son actividades económicas que producen menos empleos en relación a las demás actividades como el transporte, el comercio y los servicios. Existe una gran diferencia entre sus salarios reales. El Gráfico 6, presenta que la explotación de minas y canteras para el año 2012 tenía 298 empleos, los salarios reales per cápita en promedio eran de $\$ 1.405 .887$, mientas que en la agricultura, los salarios reales $\$ 493.157$ y se produjeron 730 empleos. 
Gráfico 6, Relación entre los salarios reales per cápita y el empleo por rama de actividad económica en San José de Cúcuta

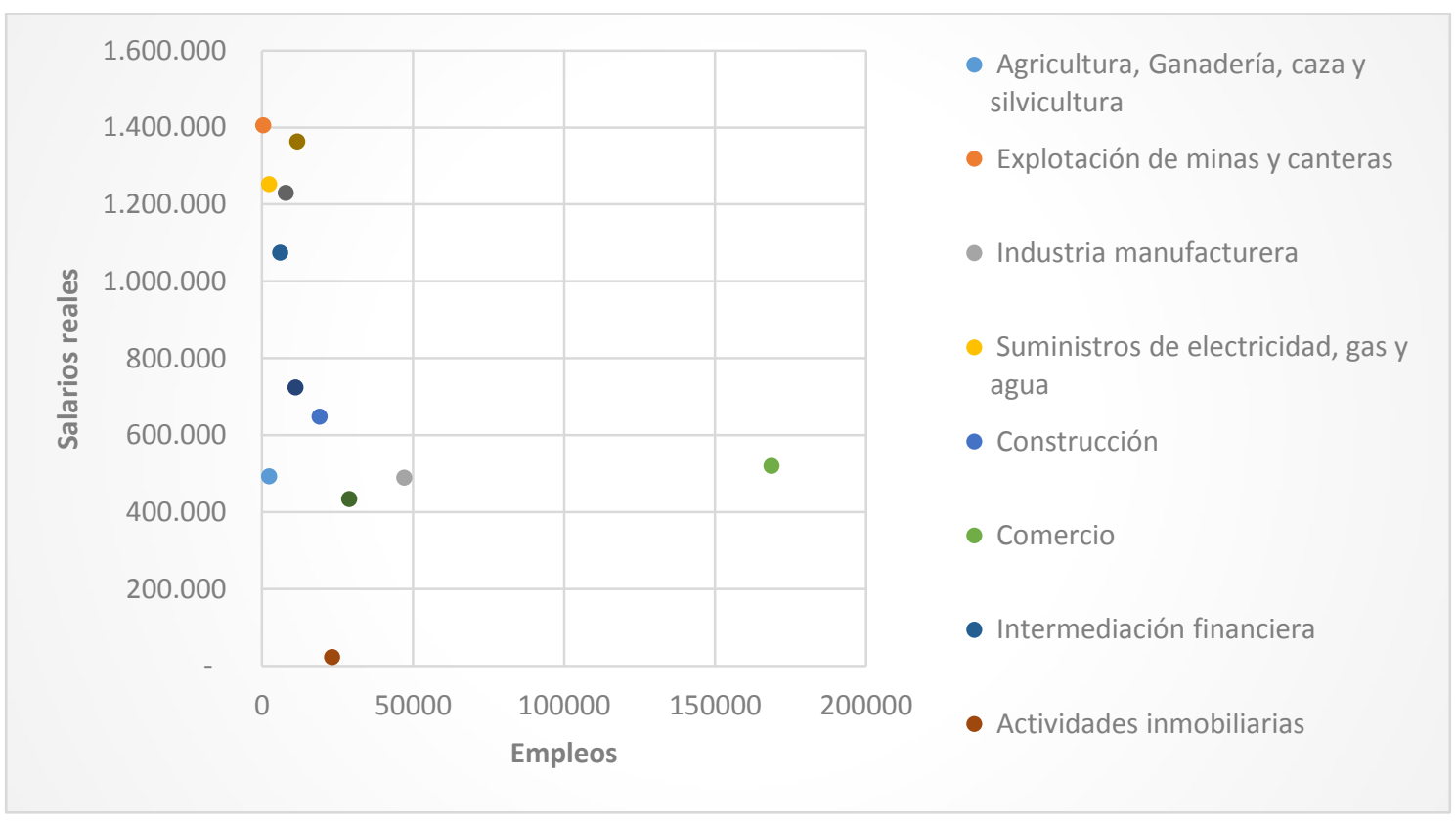

Fuente: DANE, GEIH 2012, diseño autor

El

Gráfico 7, presenta en orden las actividades que presentan mayores salarios reales per cápita en el año 2012, donde la primera actividad con mayores salarios es la explotación de minas y canteras, seguido de educación. En el caso puntual de la agricultura, ganadería, casa y silvicultura se encuentra ocupa el noveno lugar en orden de salarios per cápita.

\section{Gráfico 7 salarios reales por rama de actividad económica en San José de Cúcuta}




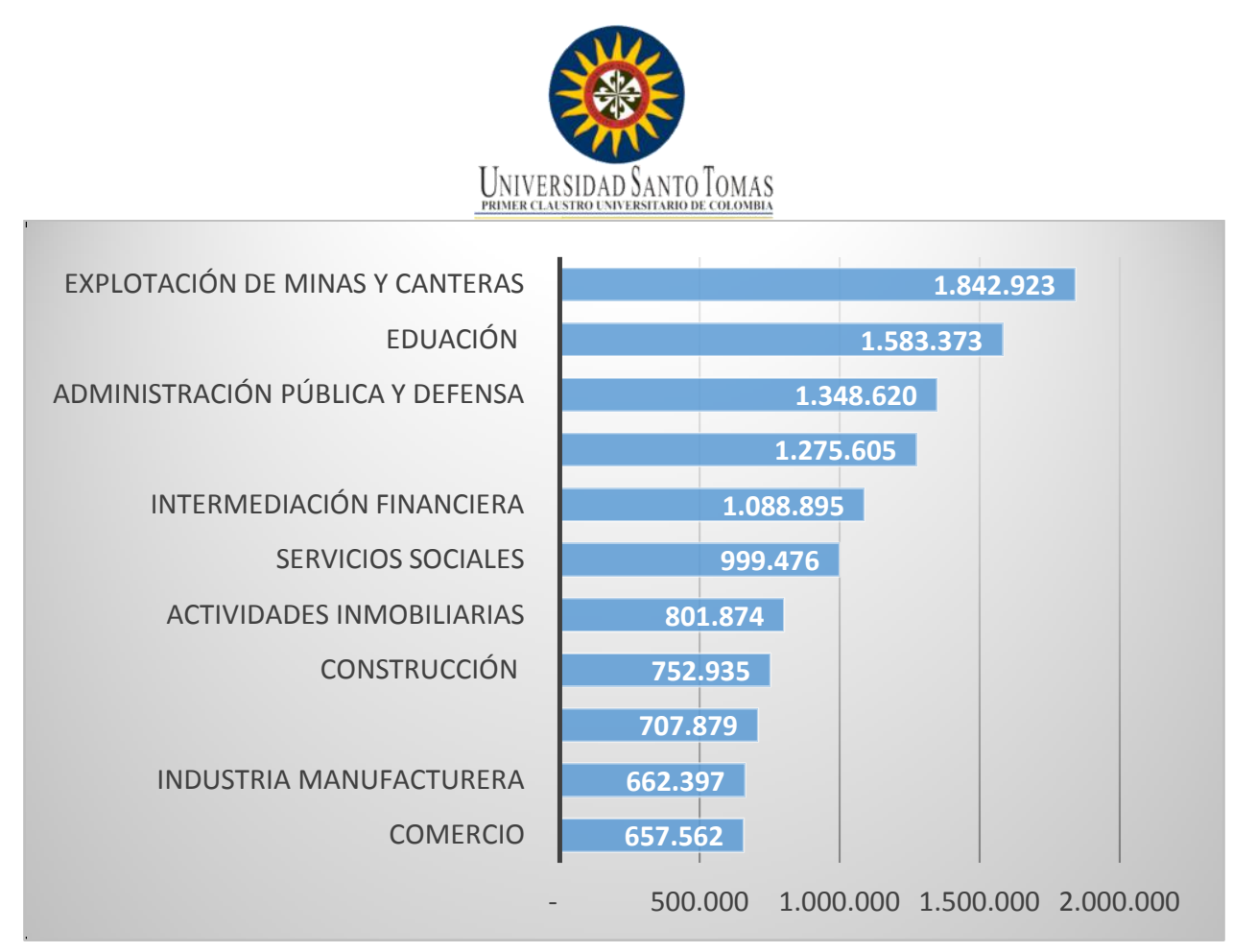

Fuente: DANE: GEIH 2012, diseño propio

En cuanto al empleo en San José de Cúcuta, se puede evidenciar de la cantidad de empleos por actividades económicas como el comercio y la industria manufacturera, los cuales producen los mayores empleos; sin embargo, producen los menores salarios como se observa en el

\section{Gráfico 7.}

Aunque las actividades de explotación de minas y canterás, produce los mayores salarios paradójicamente generan la menor cantidad de empleos en la zona. Asimismo, se evidencia que las actividades agropecuarias según el PDM 2012-2015 de San José de Cúcuta, poco a poco han sido abandonadas, dado la deficiente calidad de las tierras y el auge de las actividades mineras que poco a poco han ido erosionando las tierras cultivables, haciendo que las actividades agropecuarias pierdan valor en el mercado.

A pesar que las actividades mineras han tenido un auge en términos de salarios reales per cápita a partir de 2007, no siempre ha sido así. La Tabla 2 y el 
Gráfico 8 presentan la evolución de los salarios per cápita de los trabajadores vinculados a las actividades mineras y agropecuarias; se puede observar que los salarios en el periodo 2002-2007, en las actividades en mención, tienen unos salarios en rangos de $\$ 300.000$ a $\$ 800.000$. Sin embargo, en el periodo 2008-2012 los salarios de las actividades mineras son mucho mayores que aquellos producidos en las actividades agropecuarias.

Tabla 2 Salarios reales por actividades económicas 2002-2012

\begin{tabular}{|c|c|c|c|c|c|c|c|c|c|c|c|c|c|c|c|c|c|c|c|c|c|c|}
\hline $\begin{array}{l}\text { RAMA DE } \\
\text { ACTIVIDAD }\end{array}$ & & 2002 & & 2003 & & 2004 & & 2005 & & 2006 & & 2007 & & 2008 & & 2009 & & 2010 & & 2011 & & 2012 \\
\hline AGROPECUARIA & $\$$ & 368.293 & $\$$ & 325.681 & $\$$ & 377.425 & $\$$ & 340.160 & $\$$ & 392.323 & $\$$ & 308.040 & $\$$ & 376.677 & $\$$ & 248.161 & $\$$ & 213.098 & $\$$ & 205.444 & $\$$ & 349.160 \\
\hline MINAS Y CANTERAS & $\$$ & 284.945 & $\$$ & 615.819 & $\$$ & 524.993 & $\$$ & 528.514 & $\$$ & 621.959 & $\$$ & 478.609 & $\$$ & 1.207 .795 & $\$$ & 1.421 .090 & $\$$ & 782.816 & $\$$ & 754.698 & $\$$ & 1.780 .678 \\
\hline $\begin{array}{l}\text { INDUSTRIA } \\
\text { MANUFACTURERA }\end{array}$ & $\$$ & 342.973 & $\$$ & 424.430 & $\$$ & 339.689 & $\$$ & 387.929 & $\$$ & 435.335 & $\$$ & 351.299 & $\$$ & 533.662 & $\$$ & 321.527 & $\$$ & 276.105 & $\$$ & 266.187 & $\$$ & 422.411 \\
\hline $\begin{array}{l}\text { ELECTRICIDAD GAS } \\
\text { Y AGUA }\end{array}$ & $\$$ & 566.390 & $\$$ & 992.405 & $\$$ & 1.784 .560 & $\$$ & 1.032 .842 & $\$$ & 735.204 & $\$$ & 935.317 & $\$$ & 839.307 & $\$$ & 1.203 .832 & $\$$ & 719.299 & $\$$ & 693.462 & $\$$ & 1.143 .670 \\
\hline CONSTRUCCIÓN & $\$$ & 295.833 & $\$$ & 447.685 & $\$$ & 393.838 & $\$$ & 359.501 & $\$$ & 456.498 & $\$$ & 325.555 & $\$$ & 714.471 & $\$$ & 319.920 & $\$$ & 268.992 & $\$$ & 259.330 & $\$$ & 318.565 \\
\hline $\begin{array}{l}\text { COMERCIO } \\
\text { RESTAURANTE } \\
\text { HOTELES }\end{array}$ & $\$$ & 364.511 & $\$$ & 404.924 & $\$$ & 381.767 & $\$$ & 382.928 & $\$$ & 426.954 & $\$$ & 346.770 & $\$$ & 502.419 & $\$$ & 192.074 & $\$$ & 180.743 & $\$$ & 174.251 & $\$$ & 237.102 \\
\hline TRANSPORTE & $\$$ & 520.270 & $\$$ & 530.613 & $\$$ & 510.856 & $\$$ & 528.353 & $\$$ & 552.168 & $\$$ & 478.464 & $\$$ & 586.749 & $\$$ & 150.218 & $\$$ & 102.116 & $\$$ & 98.448 & $\$$ & 283.280 \\
\hline $\begin{array}{l}\text { ESTABLECIMIENTOS } \\
\text { FINANCIEROS }\end{array}$ & $\$$ & 620.463 & $\$$ & 889.142 & $\$$ & 697.029 & $\$$ & 838.634 & $\$$ & 798.515 & $\$$ & 759.447 & $\$$ & 814.217 & $\$$ & 753.163 & $\$$ & 658.577 & $\$$ & 634.921 & $\$$ & 1.130 .337 \\
\hline $\begin{array}{l}\text { ACTIVIDAD } \\
\text { INMOVILIARIA }\end{array}$ & $\$$ & 440.052 & $\$$ & 464.055 & $\$$ & 611.295 & $\$$ & 504.461 & $\$$ & 628.720 & $\$$ & 456.828 & $\$$ & 635.520 & $\$$ & 504.892 & $\$$ & 594.791 & $\$$ & 573.426 & $\$$ & 561.945 \\
\hline $\begin{array}{l}\text { SERVICIO } \\
\text { COMUNITARIO }\end{array}$ & & 1.067 .793 & $\$$ & 657.429 & $\$$ & 620.141 & $\$$ & 621.198 & $\$$ & 743.971 & $\$$ & 562.542 & $\$$ & 805.967 & $\$$ & 594.420 & $\$$ & 501.310 & $\$$ & 483.303 & $\$$ & 575.186 \\
\hline Total & $\$$ & 487.152 & $\$$ & 516.089 & $\$$ & 491.210 & $\$$ & 477.571 & $\$$ & 544.870 & $\$$ & 432.477 & $\$$ & 648.655 & $\$$ & 338.567 & $\$$ & 289.041 & $\$$ & 278.659 & $\$$ & 405.853 \\
\hline
\end{tabular}

Fuente: DANE, GEIH 2002-2012, diseño propio 
Gráfico 8, salarios per cápita del sector minero y agropecuario 2002-2012

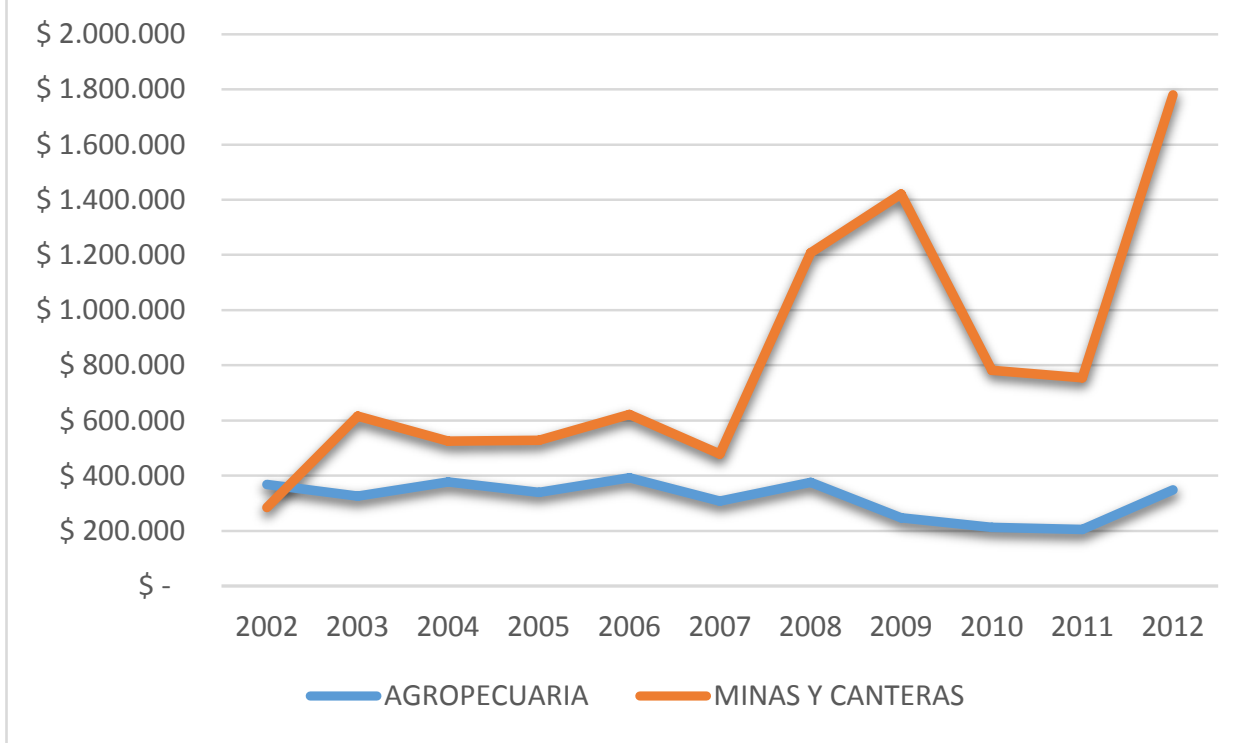

Fuente: DANE, GEIH 2002-2012, diseño propio

Mientras el sector minero presenta un auge en salarios reales per cápita, el sector agropecuario ha tenido un deterioro a partir del año 2008. En este sentido, la relación que existe entre las actividades mineras y agropecuarias, según la matriz de correlación, se presenta una incidencia negativa. Lo que demuestra que existe una relación inversa entre los dos sectores. En la medida que aumenta el sector minero, las actividades agropecuarias se reducen en -0.0014 (ver Tabla 3). Relación aunque pequeña, existe una presencia de un auge de un sector en reducción al otro.

Tabla 3, Matriz de correlación con significancia estadística

\begin{tabular}{|l|r|r|}
\hline Actividad & Agropecuario & Minas \\
\hline Agropecuario & 1 & $-0,0014$ \\
\hline significancia & & 0,9157 \\
\hline & & \\
\hline
\end{tabular}




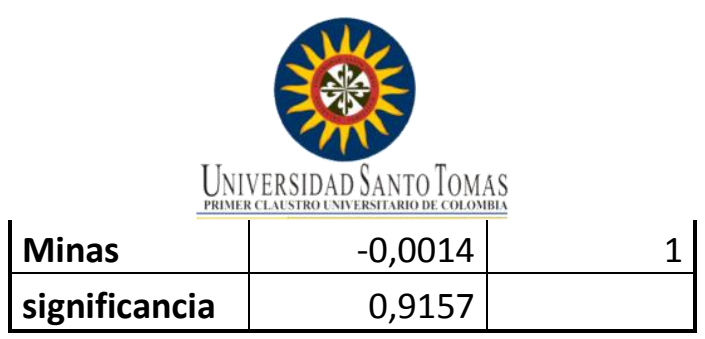

Fuente: DANE, GEIH primer semestre de 2012

El nivel de significancia de 0,9557 , lo que se puede inferir, que las actividades mineras no presentan problemas de colinealidad con las actividades agropecuarias como lo muestra la Tabla 3.

Por tanto, de acuerdo con lo descrito en el capítulo 2, las actividades mineras han presentado un auge el cual a causa un efecto negativo principalmente en los salarios de las actividades agropecuarias. Lo que afirma que las actividades como la agricultura, ganadería, silvicultura etc., no son tan bien valoradas como las actividades mineras en el municipio de San José de Cúcuta.

\subsection{ESTIMACIÓN ECONOMÉTRICA.}

La metodología que se empleará en este trabajo, para contestar a la pregunta de la investigación, consiste en la utilización de las técnicas de evaluación econométrica, con el fin de analizar el auge del ingreso real per cápita del sector minero y sus efectos en los salarios per cápita del sector agrícola, donde se estimarán modelos de corte transversal como Mínimos Cuadrados Ordinarios-MCO, donde se observará la elasticidad de los salarios reales a precios 2008 del sector minero y agropecuario, en función de variables explicativas. Asimismo, se estimará un modelo de regresión cuantilica, donde se busca presentar el cuantil que explica los salarios per cápita de la mano de obra en los sectores en mención. 


\subsection{MODELO DE MÍNIMOS CUADRADOS ORDINARIOS}

En este aparte se analizará los salarios totales de la mano de obra en las distintas actividades económicas por medio de la estimación de un modelo econométrico, conocido en la literatura como Mínimos Cuadrados Ordinarios (MCO), tomando como base la información secundaria de la Gran Encuesta Integrada de Hogares (GEIH) del año 2012.

Asimismo, se busca observar las diferencias estimadas de los salarios por cada actividad económica puntualizando a la minería y a la agricultura, en donde el salario está en función de la mano de obra de las distintas actividades, del siguiente modo:

$$
w=f(\text { agro }, \text { ind }, \text { com }, \text { cons }, \text { finan }, \text { sum }, \text { minas })
$$

Por el cual se estima por el siguiente modelo lineal:

ingresos totales

$$
\begin{aligned}
& =\beta_{0}+\beta_{1} \text { agro }+\beta_{1} \text { ind }+\beta_{2} \text { com }+\beta_{3} \text { cons }+\beta_{4} \text { finan }+\beta_{5} \text { sum }+\beta_{6} \text { minas } \\
& +e
\end{aligned}
$$

Donde las variables independientes son: agro que es la fuerza laboral del sector agropecuario, ind aquellos que pertenecen a la industria, com es el sector comercio, cons es la actividad de construcción, finan toda la mano de obra de las finanzas de la zona, sum es el suministro de electricidad, gas y agua y minas es el personal ocupado en explotación de minas y canteras. Finalmente, la variable dependiente salarios totales es el logaritmo natural del ingreso total, donde se toma el periodo de referencia del primer semestre de 2012, para el municipio de San José de Cúcuta.

Los vectores de parámetros $\beta_{1} \ldots \beta_{n}$, permiten establecer el valor esperado de la variable explicada o dependiente (salarios totales) con relación a las variables independientes 
(agropecuario, industria, comercio etc.). Igualmente, el vector $\beta_{0}$ determina cuál es el ingreso mínimo por el cual los trabajadores de San José de Cúcuta están dispuestos a trabajar.

Para ejemplarizar lo descrito en el párrafo anterior, el modelo de regresión lineal expresa la relación que existe entre la variable a explicar o dependiente (Y) que en nuestro caso son los salarios totales, y una variable explicativa o independiente $(\mathrm{X})$, donde se encuentran las ramas de actividad económica, en donde se simplifica en la siguiente expresión:

$$
Y=\beta_{0}+\beta_{1} X_{1}+e
$$

Igualmente, e representa el término de error o variable estocástica (inobservable) y $\beta_{0} y \beta_{1}$ los parámetros poblacionales (desconocidos) ya mencionados. e representa todos los factores o efectos externos que no se incorporan en el modelo que afectan a Y, además de $\mathrm{X}$. En este modelo simple suponemos que todos esos factores son inobservables. Si los factores de e se mantienen fijos, $E(e)=0$, entonces $\mathrm{X}$ tiene un efecto lineal sobre $\mathrm{Y}$

$$
Y: \Delta Y=\beta_{1} \Delta X_{1}
$$

Entonces el término de error (e) puede recoger factores inobservables como el mercado externo, los precios, la devaluación de la moneda entre otros. Bajo ciertos supuestos podremos capturar el efecto, Ceteris Paribus, de la rama de actividad económica sobre el ingreso total.

Si los factores de e se mantienen fijos, $\Delta \mathrm{e}=0$, entonces el efecto de un trabajador más sobre el ingreso total está dado por $\beta_{1}$. Para simplificar se asume que el término del error es la capacidad potencial. Por tanto, la pendiente del modelo es:

$$
\beta_{1}=\frac{\Delta E(Y \mid X)}{\Delta X}
$$


Cuando $\mathrm{X}$ cambia en una unidad adicional, $\mathrm{Y}$ varía en promedio $\beta_{1}$ unidades. La constante $\beta_{0}$ es el valor promedio de $\mathrm{Y}$ cuando $\mathrm{X}=0$ : donde el valor esperado $\mathrm{E}(\mathrm{Y} \mid \mathrm{X}=0)$. Asimismo, se observa que el modelo empleado es un modelo semielastico donde solo la variable w se linealiza por medio del logaritmo natural, lo cual la interpretación teórica es la siguiente:

$$
\begin{gathered}
E(\ln Y \mid X)=\beta_{0}+\beta_{1} X \\
\beta_{1}=\frac{\Delta E(\ln Y \mid X)}{\Delta X} \approx \frac{\Delta E(Y \mid X) / \Delta E(Y \mid X)}{\Delta X}
\end{gathered}
$$

Para expresar la variación de Y en términos porcentuales se multiplica por 100

$$
100 \beta_{1}=\frac{E((100 x \Delta Y / Y \mid X)}{\Delta X}
$$

Cuando X cambia una unidad adicional, Y cambia en promedio $100 \beta_{1} \%$. Sin embargo, dado que resultados del modelo se obtuvieron en valores absolutos, se tienen que los salarios totales estimados por un aumento de un trabajador adicional correspondiente a cada rama de actividad económica en promedio, se encuentran en promedio entre menos y un salario mínimo legal en el periodo estudiado como lo presenta la Tabla 4. 
Tabla 4, Regresión por Mínimos Cuadrados Ordinarios (MCO) de los salarios totales correspondientes a las ramas de actividad económica de San José de Cúcuta.

\begin{tabular}{|c|c|c|c|c|c|}
\hline & & & & & $\begin{array}{c}\text { Número de of observaciones = } \\
5.736\end{array}$ \\
\hline & & & & & $F(7, \mathbf{5 7 2 8})=159.42$ \\
\hline & & & & & Prob $>F=0.0000$ \\
\hline & & & & & R-cuadrado $=0.1631$ \\
\hline & & & & & $\mathrm{R}$ cuadrado ajustado $=0.1620$ \\
\hline & & & & & Root MSE $=5.0 \mathrm{e}+05$ \\
\hline salarios totales & Cor & ficientes & $T$ & $P>|t|$ & [95\% Conf. Interval] \\
\hline agropecuario & $\$$ & 456.619 & 2.71 & 0.007 & $93871.16 \quad 586849$ \\
\hline Industria & $\$$ & 490.125 & 13.18 & 0.000 & \begin{tabular}{|ll}
$318240.6 \quad 429492.8$ \\
\end{tabular} \\
\hline Comercio & $\$$ & 544.283 & 26.22 & 0.000 & $396028.2 \quad 460020.2$ \\
\hline Construcción & $\$$ & 664.789 & 12.66 & 0.000 & $463607.5 \quad 633453.5$ \\
\hline Financiera & $\$$ & 1.100 .983 & 13.37 & 0.000 & $840343.2 \quad 1129105$ \\
\hline Suministro & $\$$ & 1.261 .700 & 10.18 & 0.000 & $924863 \quad 1366020$ \\
\hline Minas & $\$$ & 1.340 .000 & 4.87 & 0.000 & $731514.8 \quad 1715968$ \\
\hline Constante & $\$$ & $116.258,70$ & 14.51 & 0.000 & \begin{tabular}{|ll}
100551.1 & 131966.3
\end{tabular} \\
\hline
\end{tabular}

Fuente: DANE, GEIH 2012, cálculos Stata 12

Dado que se tiene 5.736 observaciones en la base de datos de GEIH para el municipio de San José de Cúcuta, donde se observa que en la casilla de la Tabla 4 ;Error! No se encuentra el origen de la referencia. los coeficientes que muestran el valor de cada $\beta_{1} \ldots . \beta_{7}$, donde las actividades agropecuarias poseen los menores salarios promedio con respecto a los demás sectores como suministro de gas y electricidad y la minería, como se evidencia en la Gráfico 9. 
Gráfico 9, Estimación de los salarios reales con respecto a las actividades económicas

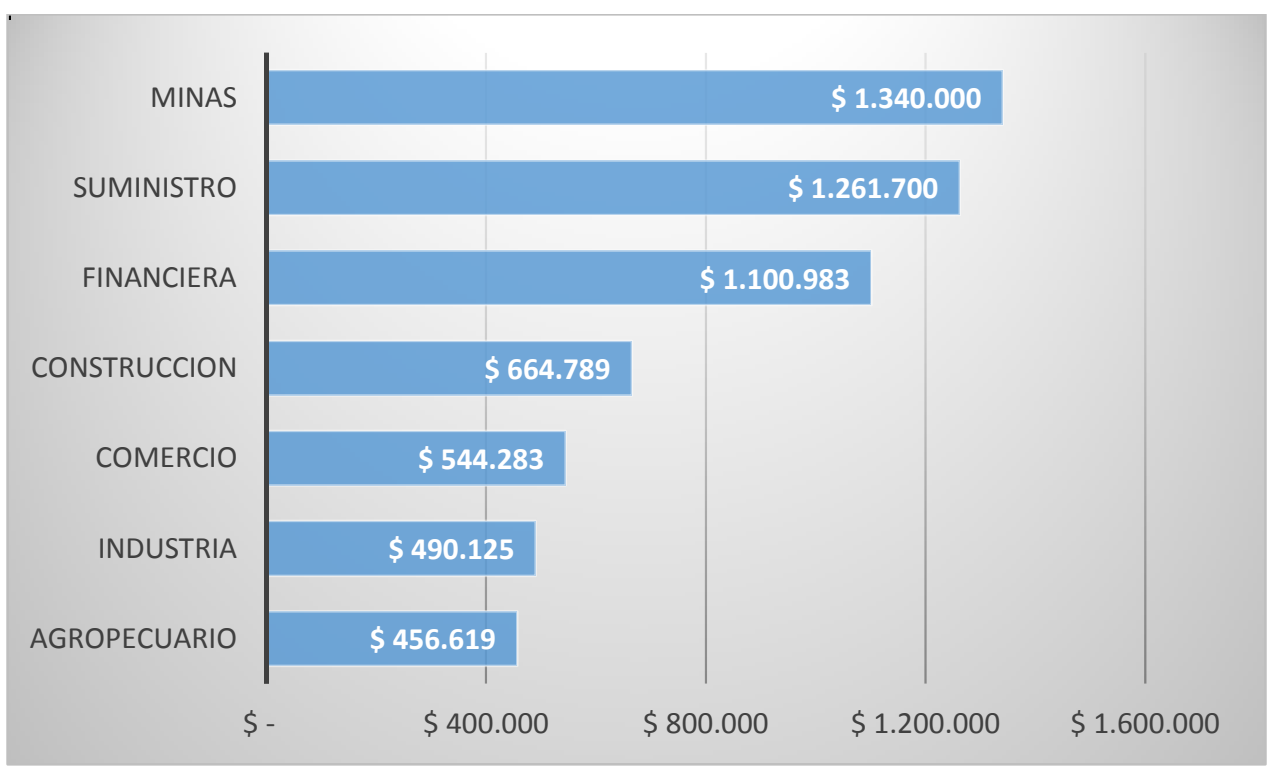

Fuente: DANE, GEIH primer semestre de 2012

De otra parte, se puede observar en la Tabla 4, que el R cuadrado y el R ajustado arrojan el valor de 0,1631 y 0,1620 respectivamente. Principalmente, por los k grados de libertad y el tamaño de muestra grande, produce que la bondad de ajuste $\mathrm{R}^{2}$ se reduzca, dado que es un indicador sensible ante los posibles aumentos de variables y tamaños de muestra del modelo.

De otra parte, los otros sectores como el comercio la industria y la construcción, presentan mayores salarios per cápita con respecto a la agricultura. Estos también pueden ser mayormente valorados. De hecho, el comercio es la principal actividad que más produce empleos.

Sin embargo, el comercio, la construcción y la industria se desarrollan en el área metropolitana, las mineras y agropecuarias son explotadas en las zonas rurales, lo que permite que ambas utilicen la tierra como principal variable para la producción. En la medida que se expanda la minería en hectáreas de tierra, la agricultura se reduciría (como se evidenció en el capitulo 2) 


\section{(1) \\ $\left.\frac{1}{4}\right)^{3}$ \\ Universidad Santo Tomas \\ 3.4. MODELO DE REGRESIONES CUANTILICAS DEL INGRESO DE LOS SECTORES ECONÓMICOS DE SAN JOSÉ DE CÚCUTA}

Para completar la investigación En este estudio se analiza el efecto de las actividades económicas sobre los salarios reales que refleja los resultados de esta investigación. Asimismo, este modelo que además es no paramétrico es el método que estima los regresores conocidos como $\beta_{1}, \ldots \beta_{n}$, para cada cuantil de salario real.

El objetivo fundamental es estimar la función de m en la ecuación de regresión de la forma

$$
y_{i}=m\left(x_{i}\right)+e_{i}
$$

Donde $x_{i}$ es la variable explicativa o independiente e "i" es una media de cero residual, que se supone independiente de $\left(x_{i}\right)$. Por otro lado, la función de $m$ es la estimación puntual. Según Måns Söderbom (2013), este enfoque, se basa en cuanto varía $y_{i}$ ante cambios en $x_{i}$.

Asimismo, el principal objeto del análisis de regresión cuantílica es determinar la relación entre una variable respuesta (salarios) y un conjunto de variables predictoras (agropecuarios, minas y canteras, comercio, industria manufacturera etc.). López y Mora (2007) exponen que en los modelos de regresión cuantílica, los errores siguen procesos de sucesión $u_{i}, \ldots u_{n}$ de variables aleatorias o estocásticas, independientes e idénticamente distribuidas (iid) con media cero $\left(E\left(u_{n}=0\right)\right.$ y varianza constante $\sigma^{2}$, asumen que la distribución generalmente es la normal.

Por tanto, Koenker \& Bassett (1978) introducen el concepto de regresión cuantílica (RC) como una solución a los problemas de no normalidad en el diseño experimental en una muestra de corte trnasversal y demuestran que los estimadores por cuantiles son más eficientes que el estimador máximo verosímil de muchos modelos paramétricos convencionales. 


\section{M.

De otra parte, Vélez (2011), la medida de tendencia central, (en este caso la mediana) es un caso particular de cuantil ( $\tau=0,5$ ) de las regresiones cuantilicas. En términos generales, la relación entre una variable respuesta y un grupo de variables predictoras puede ser explorada para diversos cuantiles, lo que permite conocer mejor los cambios que ocurren en la respuesta de acuerdo a los cambios de las variables predictoras.

Para estimar el modelo de regresión cuantilica, se utilizará el esquema propuesto por López \& Mora (2007) tendremos la los salarios reales como una variable aleatoria real. Dado un $\tau \in(0,1)$ y una variable aleatoria $l_{-} y$ (discreta), el $\tau$-ésimo cuantil es definido como:

$$
Q(\tau)=\inf \left\{\ln \_y: F\left(\ln \_y\right) \geq \tau\right\}
$$

donde F es la función de distribución de AC

Por otro lado, si se tiene $\left\{\ln \_y 1, \ln \_y 2, \ldots, \ln \_y_{\mathrm{n}}\right\}$, se encuentra la estimación de la función de distribución, por medio de la muestra experimental que se obtuvo en campo la cual la muestra definida como el cociente entre el número de las observaciones inferiores o iguales al valor de interés y el número total de las observaciones:

$$
\hat{F}\left(\ln _{-} y\right)=\frac{\#\left(\ln _{y} \quad i \leq \ln \_y\right)}{n}
$$

Donde la fucnción de la ln_y es igual el numero de observaciones menor o igual a la vairable continua de la cción colectiva, sobre el numero total de observaciones, por tanto el cálculo de cuantil es:

$$
\hat{Q}(\tau)=\underset{\varepsilon_{\tau} \in R}{\operatorname{argmin}}\left\{\sum_{y_{i} \geq \varepsilon_{\tau}} \tau\left|\ln { }_{-} y_{i}-\varepsilon_{\tau}\right|+\sum_{y_{i}}(1-\tau)\left|y_{i}-\varepsilon_{\tau}\right|\right\}
$$


De otra parte se estima el modelo de regresiones cuantilicas del siguiente modo:

$$
\begin{aligned}
\ln \_y=\beta_{0}+ & \beta_{1} \text { agro }+\beta_{2} \text { minas }+\beta_{3} \text { ind }+\beta_{4} \text { sum }+\beta_{5} \text { con }+\beta_{6} \text { com }+\beta_{7} \text { fin } \\
& +\beta_{8} \text { inm }+e
\end{aligned}
$$

Donde el término del error satisface $E\left(\ln _{-} y \mid X_{i}\right)=0, X_{i}$ representa las variables independientes. Se denota el qth la función del cuantil condicional de ln_y dada las x variables independientes $Q_{q}$. En general la formalización del modelo se define del siguiente modo:

$$
\begin{aligned}
Q_{q}\left(\ln \_y \mid X_{i}\right) & =\beta_{0}+\beta_{1} \text { agro }+\beta_{2} \text { minas }+\beta_{3} \text { ind }+\beta_{4} \text { sum }+\beta_{5} \text { con }+\beta_{6} \text { com } \\
& +\beta_{7} \text { fin }+\beta_{8} \text { inm }+F_{u_{i}}^{-1}(q)
\end{aligned}
$$

Donde $F_{u_{i}}$ es la función de distribución de $u_{i}$. El cuantil depende de la distribución de $u_{i}$ vía al término de $F_{u_{i}}^{-1}(q)$. Esto dependerá de $x_{i}$ sí, por ejemplo, los errores son heterosdasticos. Entonces, en general, $Q_{q}\left(\ln _{-} y \mid X_{i}\right)$ valores en diferencia de q diferirá en algo más que el intercepto el cual es incluso no lineal.

En el caso especial de que los errores son iid, ocurre una simplificación de $F_{u_{i}}^{-1}(q)=$ $F_{u}^{-1}(q)$, el cual no varía con i. Entonces los cuantiles condicionales son:

$$
Q_{q}\left(\ln _{-} y \mid X_{i}\right)=\left\{\beta_{1}+F_{u}^{-1}(q)\right\}+\beta_{2}+x_{i}
$$

La función condicional de los cuantiles tienen una pendiente común, diferenciando solo en el intercepto $\beta_{0}+F_{u}^{-1}(q)$. Por otro lado los erreores no son iid, por la Heteroscedasticidad. El enfoque de cuantil estándar especifica la función condicional de los cuantiles y puede ser expresada como un modelo de MCO, a través de la matriz boostraping, donde la pendiente del modelo es el siguiente: 


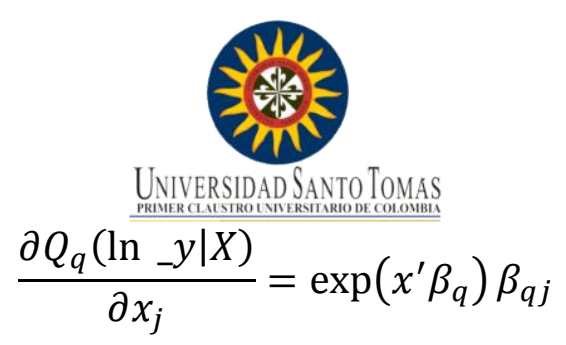

El cuantil 0.5 especifica la estimación y deben contener números entre 0 y 1 , exclusivo. Números mayores que 1 se interpretan como porcentajes. El valor por defecto de 0,5 corresponde a la mediana, por tanto el modelo de regresión que se obtuvo es el siguiente:

$Q_{q}\left(\ln \_y \mid X_{i}\right)=\beta_{0}+\beta_{1}$ agro $+\beta_{2}$ minas $+\beta_{3}$ ind $+\beta_{4}$ sum $+\beta_{5}$ con $+\beta_{6}$ com + $\beta_{7}$ fin $+\beta_{8}$ inm $+\beta_{9}$ def $+\beta_{10} e d u+F_{u_{i}}^{-1}(q)$, quantile (0.5) repeticiones (100)

El resultado obtenido se refleja en la Tabla 5, donde se estimaron los años 2002, 2006, 2010 y 2012, de las diferentes ramas de actividad económica de San José de Cúcuta. Sin embargo, solo el análisis se centrará en la agricultura y a minería dado que son el objeto de la investigación.

Tabla 5, Resultados de la regresión cuantilica para el logaritmo natural del salario y las ramas de actividad económica

\begin{tabular}{|l|r|r|r|r|}
\hline q50 & \multicolumn{1}{|l|}{2002} & \multicolumn{1}{l|}{2006} & \multicolumn{1}{l|}{2010} & \multicolumn{1}{l|}{ Coeficientes } \\
\hline Actividades & \multicolumn{4}{|c|}{} \\
\hline Agropecuario & $-0,2231436$ & $-0,2639008$ & $-0,2624474$ & 0,1335306 \\
\hline minas & 0,0295582 & 0,00 & 0,4054651 & 1,272965 \\
\hline industria & $-0,0689926$ & $-0,1303701$ & $-0,2624474$ & 0,3015137 \\
\hline electricidad & 0,7576857 & 0,0927734 & $-0,1431007$ & 0,9019012 \\
\hline construcción & $-0,0689926$ & $-0,1303701$ & $-0,1823215$ & 0,5108252 \\
\hline transporte & 0,0295582 & $-0,1303701$ & $-0,2624474$ & 0,322773 \\
\hline financieros & 0,1339474 & 0,00 & $-0,1823215$ & 0,5389957 \\
\hline inmobi7iaria & 0,2876816 & 0,0927734 & 0,00 & 0,3566742 \\
\hline -cons & 12,61154 & 13,02959 & 13,30468 & 12,94801 \\
\hline
\end{tabular}

Fuente: DANE, ECH 2002-2006 y GEIH 2010-2012

Como se puede observar en Gráfico 10 y en la Tabla 5, el cuantil 0.5 presenta que un empleo adicional en las actividades agropecuarias, en los años 2002, 2006, 2010, 2012, en 
UnIVERSIDAD SANT0 Tomas

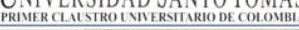

donde las actividades agropecuarias han presentado disminuciones del salarios per cápita $-0,22$ en el año 2002, -0,26 en el año 2006 y 2010 y tan solo aumento 0,13 puntos en el año 2012.

\section{Gráfico 10, Resultados de la regresión cuantilica para el logaritmo natural del salario y las ramas de actividad económica}

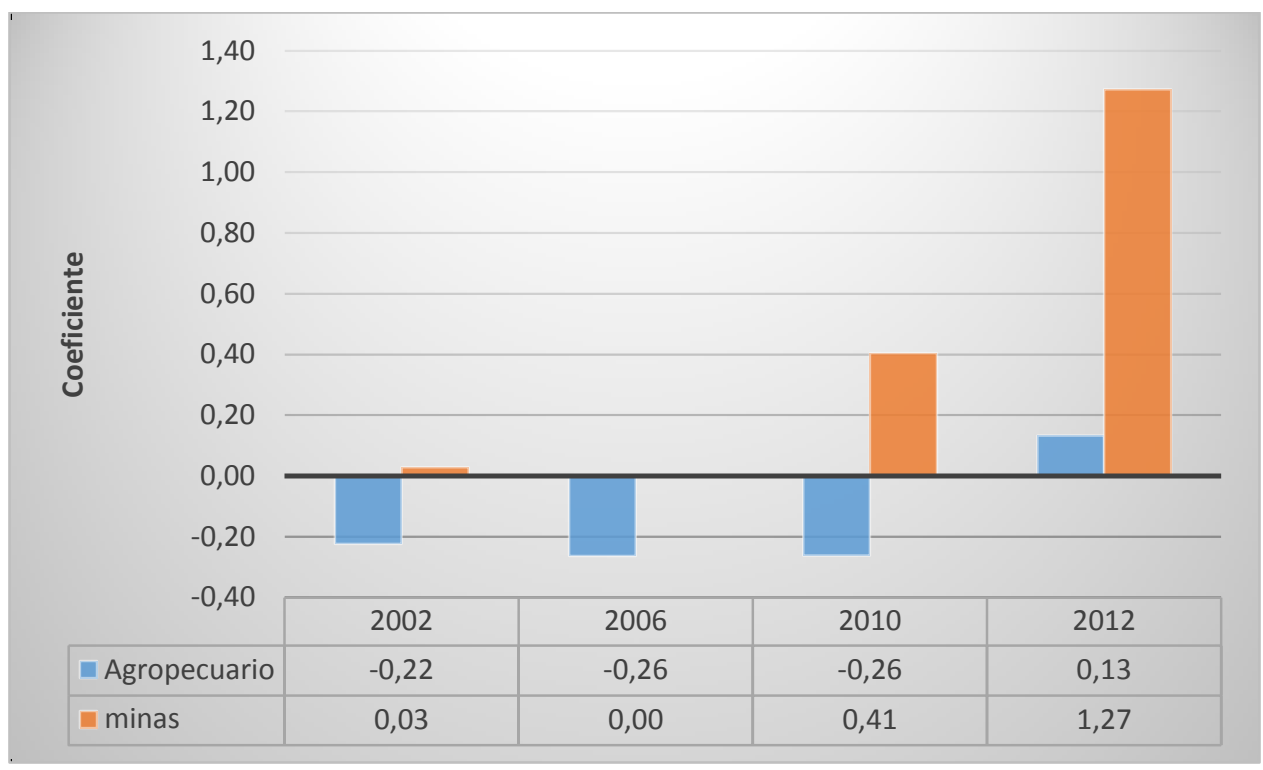

Fuente: DANE, GEIH 2002-2012, diseño propio

De igual manera, se puede evidenciar que, los resultados de los coeficientes en el año 2002, 2006 y 2010 son negativos para el sector agrario, en cuanto sus salarios per cápita. Principalmente, se debe a los bajos salarios reales que existen en el sector en mención y a la reducción de empleo que se presenta en esos periodos.

Las actividades mineras han tenido un aumento significativo principalmente en los años 2010 y 2012, donde un empleo adicional, ha generado aumentos del ingreso real per cápita de 0,40 y 1,27 respectivamente (ceteris paribus) 


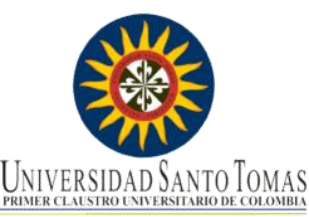

De otra parte, el signo positivo en los coeficientes de 2012, significa un aumento del salario per cápita real, con un aumento en el empleo en el primer sector de la economía. En cuanto a al sector minero, los resultados fueron positivos, pero se evidencia que el coeficiente para el año 2012 obtuvo un resultado de 1,27. Como se evidenció en los gráfico 5 y 8, tanto los salarios reales pércapita y empleo tuvieron un crecimiento mucho mayor que los años anteriores.

Asimismo, de acuerdo con el efecto que produce las actividades mineras en el uso del suelo, la evidencia empírica y lo descritos por el PDM 2012-2015 de San José de Cúcuta, en este municipio se produce desincentivos para la inversión privada en el sector primario, debido al daño ambiental que produce las actividades mineras de carbón, en las fuentes de recursos hídricos, los cuales cambian la dinámica de aguas subterráneas, que deterioran la calidad de las tierras cultivables, lo que coadyuva a que las actividades agrícolas y pecuarias no sean tan bien valoradas como el sector minero.

Por tal razón, y de acuerdo con lo descrito en el plan de desarrollo de San José de Cúcuta, lo que ha venido evidenciándose en la movilidad laboral, es que la mano de obra valora las actividades mineras a las agrícolas y pecuarias, que permite evidenciar una vinculación al sector minero u otros sectores como el comercio, para poder capturar mayores salarios o renta, 


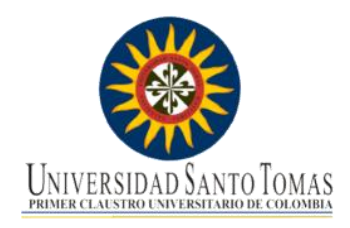

\section{CONCLUSIONES}

El sector minero en el municipio de San José de Cúcuta capital del departamento correspondiente de Norte de Santander, ha producido una reducción de las actividades agropecuarias, que según el PDM de San José de Cúcuta (2008-2011) en su página 162, arguye que las actividades agropecuarias, presenta una baja rentabilidad, produciendo de esta manera que las economías de subsistencia o campesinas se vean obligadas a abandonar sus actividades y desparecer su franja agrícola.

Esta evidencia descrita en el párrafo anterior, se observó en el capítulo II, donde se analizó el índice de GINI y la tenencia de la propiedad, y se halló que desde el año 2002 hasta el año 2010 los predios que pertenecían a microfundio, minifundios y pequeñas propiedades se redujeron drásticamente, aumentado así los predios de mediana y gran propiedad.

De otra parte se halló que las actividades mineras en especial la extracción de carbón mineral, ocupa más de 8.166 hectáreas concentradas en varias zonas rurales del municipio de San José de Cúcuta, que corresponde el $57 \%$ de las hectáreas destinadas a la explotación.

En el caso particular del empleo, la ocupación en el sector agrario presentó fluctuaciones y disminuciones en periodos de más de 4 años, como en el periodo de 2003-2008, que pasó en el año de 2003 con 1.150 empleos a 521 en 2008. En contraste, el sector minero mantuvo una tendencia constante manteniéndose en una tendencia de 300 empleos a partir del año 2003 a 2012. En este sentido, se pudo observar en el Capítulo II, que la mano de obra del sector agrario es instable, dado que no puede mantener una tendencia creciente en el periodo 2001-2012.

Los salarios reales per cápita en San José de Cúcuta, es una variable que es notoria la 
desproporción entre el sector minero y el sector agrícola, dado que si bien en las actividades del sector agrícola, hay disminución de empleos, sus salarios per capitas son muy bajo en relación a aquellos correspondientes al sector minero. Mientras que un empleado en promedio ganaba en el año $2012 \$ 349.160$, un trabajador vinculado a la minería ganaba $\$ 1.780 .678$ en el mismo año, siendo el 2012 el año con mayor aumento salarial per cápita con respecto al periodo 2002-2012.

Así, se observa que tanto la concentración de la tierra, el aumento de los grandes terratenientes, la reducción de empleos en el sector agrícola y salarios por debajo de $\$ 400.000$, produce que las actividades agropecuarias, presenten un menor valor en las economías de subsistencia o campesinas; quienes abandonaron sus actividades dándole paso en parte a las actividades mineras. 


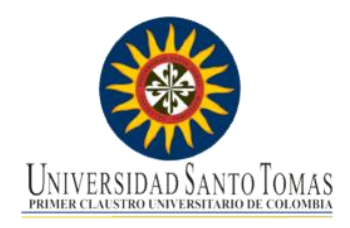

\section{BIBLIOGRAFÍA}

1) Bruce, Johnston y Mellor (1961). "El papel de la agricultura en el desarrollo económico", reproducido en Flórez E., 1972. Lecturas sobre desarrollo agrícola, Fondo de Cultura Económica, México, pp. 23-53.

2) Gouverneur J., (1978). Elementos de d'Economie Politique Marxiste, Ed. Contradictions, Bruselas.

3) Misión del Empleo, (1986). El Problema Laboral Colombiano: Diagnóstico, perspectivas y políticas. Informe final de la Misión. Economía Colombiana, separata No. 10, agosto-septiembre.

4) Moncayo V., y Rojas F., (1980). Producción Campesina y Capitalismo, Cinep, Bogotá.

5) Vargas (1987). “La economía campesina: consideraciones teóricas”, Cuadernos de Economía, Vol. VIII, No. 10, primer semestre, pp. 93-124. 


\section{Anexos}

$Q_{q}\left(\ln \_y \mid X_{i}\right)=\beta_{0}+\beta_{1}$ agro $+\beta_{2}$ minas $+\beta_{3}$ ind $+\beta_{4}$ sum $+\beta_{5}$ con $+\beta_{6}$ com + $\beta_{7}$ fin $+\beta_{8}$ inm $+\beta_{9}$ def $+\beta_{10} e d u+F_{u_{i}}^{-1}(q)$, quantile (.25.5 .75) repeticiones (100) 


\begin{tabular}{|c|c|c|c|c|c|c|}
\hline \multicolumn{4}{|c|}{$\begin{array}{l}\text { Simultaneous quantile regression } \\
\text { bootstrap (20) SES }\end{array}$} & \multicolumn{2}{|c|}{$\begin{array}{l}\text { Number of obs }= \\
.25 \text { Pseudo R2 }= \\
.50 \text { Pseudo R2 }= \\
.75 \text { Pseudo R2 }=\end{array}$} & $\begin{array}{l}2073 \\
0.0624 \\
0.0561 \\
0.0993\end{array}$ \\
\hline $1 n_{-} y$ & Coef. & $\begin{array}{l}\text { Bootstrap } \\
\text { std. Err. }\end{array}$ & $\mathrm{t}$ & $P>|t|$ & [95\% Conf. & Interva1] \\
\hline $\begin{array}{r}\text { q25 } \\
\text { agropecuario } \\
\text { minas } \\
\text { industria } \\
\text { suministro } \\
\text { construccion } \\
\text { comercio } \\
\text { financiera } \\
\text { inmobiliarias } \\
\text { defensa } \\
\text { eduacion } \\
\text {-cons }\end{array}$ & $\begin{array}{r}-.196826 \\
1.540445 \\
.2876816 \\
.8472977 \\
-6592455 \\
-1.65 \mathrm{e}-14 \\
.7259369 \\
.2876816 \\
1.147402 \\
.8663912 \\
12.61154\end{array}$ & $\begin{array}{l}.2276068 \\
.3812101 \\
.1200202 \\
.1568949 \\
.1448568 \\
.1153586 \\
.1206791 \\
.0915947 \\
.1491083 \\
.1932874 \\
.1061352\end{array}$ & $\begin{array}{r}-0.86 \\
4.04 \\
2.40 \\
5.40 \\
4.55 \\
-0.00 \\
6.02 \\
3.14 \\
7.70 \\
4.48 \\
118.83\end{array}$ & $\begin{array}{l}0.007 \\
0.000 \\
0.017 \\
0.000 \\
0.000 \\
1.000 \\
0.000 \\
0.002 \\
0.000 \\
0.000 \\
0.000\end{array}$ & $\begin{array}{r}-.6431892 \\
.7928485 \\
.0523082 \\
.5396087 \\
.3751646 \\
-.2262315 \\
.4892713 \\
.1080538 \\
.8549833 \\
.4873324 \\
12.40339\end{array}$ & $\begin{array}{r}.2495372 \\
2.288042 \\
.5230549 \\
1.154987 \\
.9433263 \\
.2262315 \\
.9626025 \\
.4673094 \\
1.43982 \\
1.24545 \\
12.81968\end{array}$ \\
\hline $\begin{array}{r}\text { q50 agropecuario } \\
\text { minas } \\
\text { industria } \\
\text { suministro } \\
\text { construccion } \\
\text { comercio } \\
\text { financiera } \\
\text { inmobiliarias } \\
\text { defensa } \\
\text { eduacion } \\
\text {-cons }\end{array}$ & $\begin{array}{l}.1335306 \\
1.272965 \\
.3015137 \\
.9019012 \\
.5108252 \\
.322773 \\
.5389957 \\
.3566742 \\
1.129865 \\
1.129865 \\
12.94801\end{array}$ & $\begin{array}{l}.322695 \\
.3527278 \\
.0922816 \\
.245019 \\
.1046221 \\
.0926799 \\
.1367327 \\
.0880927 \\
.109249 \\
.1063534 \\
.0883796\end{array}$ & $\begin{array}{r}0.41 \\
3.61 \\
3.27 \\
3.68 \\
4.88 \\
3.48 \\
3.94 \\
4.05 \\
10.34 \\
10.62 \\
146.50\end{array}$ & $\begin{array}{l}0.009 \\
0.000 \\
0.001 \\
0.000 \\
0.000 \\
0.001 \\
0.000 \\
0.000 \\
0.000 \\
0.000 \\
0.000\end{array}$ & $\begin{array}{r}-.4993115 \\
.5812257 \\
.1205389 \\
.4213907 \\
.3056491 \\
.141017 \\
.2708473 \\
.1839143 \\
.9156149 \\
.9212934 \\
12.77469\end{array}$ & $\begin{array}{r}.7663727 \\
1.964705 \\
.4824884 \\
1.382412 \\
.7160012 \\
.504529 \\
.8071442 \\
.5294341 \\
1.344114 \\
1.338436 \\
13.12133\end{array}$ \\
\hline $\begin{array}{r}\text { q75 } \\
\text { agropecuario } \\
\text { minas } \\
\text { industria } \\
\text { suministro } \\
\text { construccion } \\
\text { comercio } \\
\text { financiera } \\
\text { inmobiliarias } \\
\text { defensa } \\
\text { eduacion } \\
\text { _cons }\end{array}$ & $\begin{array}{l}.0588408 \\
1.315677 \\
.0588408 \\
.7519884 \\
.3101549 \\
.0588408 \\
.820981 \\
.1923723 \\
1.003303 \\
1.220204 \\
13.39999\end{array}$ & $\begin{array}{l}.191764 \\
.3429241 \\
.0543583 \\
.3141319 \\
.0904318 \\
.0540787 \\
.1869658 \\
.0532951 \\
.1188006 \\
.0857224 \\
.0464924\end{array}$ & $\begin{array}{r}0.31 \\
3.84 \\
1.08 \\
2.39 \\
3.43 \\
1.09 \\
4.39 \\
3.61 \\
8.45 \\
14.23 \\
288.22\end{array}$ & $\begin{array}{l}0.759 \\
0.000 \\
0.279 \\
0.017 \\
0.001 \\
0.277 \\
0.000 \\
0.000 \\
0.000 \\
0.000 \\
0.000\end{array}$ & $\begin{array}{r}-.3172306 \\
.6431631 \\
-.0477621 \\
.1359397 \\
.1328077 \\
-.0472139 \\
.4543196 \\
.0878545 \\
.7703209 \\
1.052093 \\
13.30882\end{array}$ & $\begin{array}{r}.4349121 \\
1.98819 \\
.1654436 \\
1.368037 \\
.4875021 \\
1648954 \\
1.187642 \\
.2968902 \\
1.236284 \\
1.388316 \\
13.49117\end{array}$ \\
\hline
\end{tabular}

signrank agropecuario = minas

wi1coxon signed-rank test

sign | obs sum ranks expected

$\begin{array}{rrrr}\text { positive | } & 16 & 91624 & 57265 \\ \text { negative | } & 4 & 22906 & 57265\end{array}$




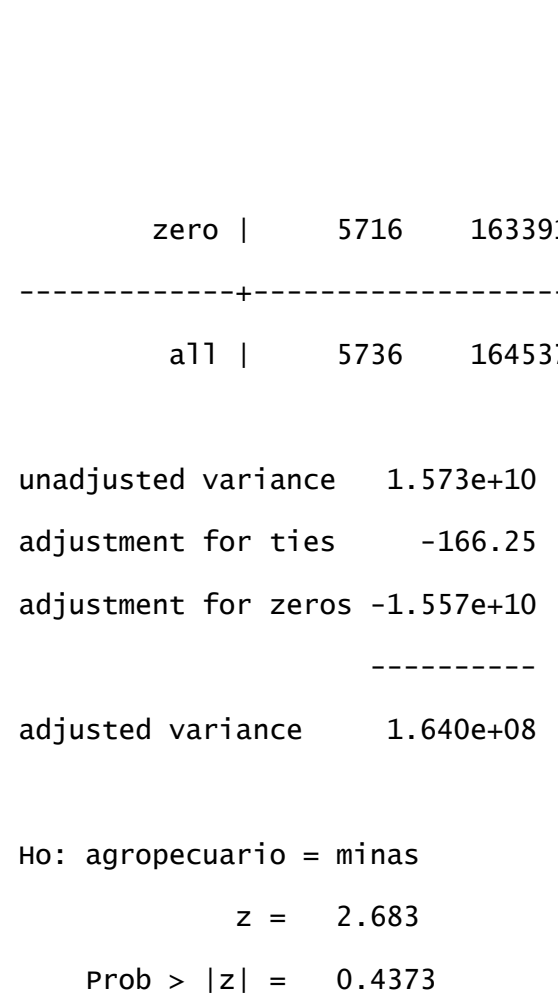


Año 2002

\begin{tabular}{|c|c|c|c|c|c|c|}
\hline & | & Bootstrap & & & & \\
\hline 1n_w & Coef. & Std. Err. & $\mathrm{t}$ & $P>|t|$ & [95\% Conf. & Interva1] \\
\hline q50 & | & & & & & \\
\hline Agropecuario & | $\quad-.2231436$ & .0604166 & -3.69 & 0.000 & -.341588 & -.1046991 \\
\hline minas & .0295582 & .0474549 & 0.62 & 0.533 & -.0634753 & .1225917 \\
\hline industria & $\mid-.0689926$ & .0222318 & -3.10 & 0.002 & -.1125773 & -.025408 \\
\hline electricidad & .7576857 & .1266575 & 5.98 & 0.000 & .5093784 & 1.005993 \\
\hline construcción & $\mid-.0689926$ & .0887816 & -0.78 & 0.437 & -.2430456 & .1050604 \\
\hline transporte & .0295582 & .0232445 & 1.27 & 0.204 & -.0160117 & .0751281 \\
\hline financieros & .1339474 & .0144646 & 9.26 & 0.000 & .1055901 & .1623047 \\
\hline inmobiliaria & .2876816 & .1101799 & 2.61 & 0.009 & .071678 & .5036851 \\
\hline _cons & 12.61154 & .0144646 & 871.89 & 0.000 & 12.58318 & 12.6399 \\
\hline
\end{tabular}

Año 2006

Simultaneous quantile regression

\begin{tabular}{|c|c|c|c|c|c|c|}
\hline & & Bootstrap & & & & \\
\hline 1n_w & Coef. & Std. Err. & $\mathrm{t}$ & $P>|t|$ & [95\% Conf. & Interva1] \\
\hline q50 & & & & & & \\
\hline Agropecuario & -.2639008 & .0412158 & -6.40 & 0.000 & -.3447199 & -.1830816 \\
\hline minas & $-1.71 e-16$ & .0497788 & -0.00 & 1.000 & -.0976101 & .0976101 \\
\hline industria & -.1303701 & $2.92 \mathrm{e}-15$ & $-4.5 e+13$ & 0.000 & -.1303701 & -.1303701 \\
\hline electricidad & .0927734 & .1185895 & 0.78 & 0.434 & -.1397661 & .325313 \\
\hline
\end{tabular}




\begin{tabular}{|c|c|c|c|c|c|c|}
\hline & & UNIVERSID, & $\begin{array}{l}\text { TTOTOMAS } \\
\text { RODECCOONBAL }\end{array}$ & & & \\
\hline construcción | & -.1303701 & .050976 & -2.56 & 0.011 & -.2303279 & -.0304124 \\
\hline transporte | & -.1303701 & .020661 & -6.31 & 0.000 & -.1708838 & -.0898565 \\
\hline financieros I & $5.07 e-15$ & .003863 & 0.00 & 1.000 & -.0075749 & .0075749 \\
\hline inmobiliaria | & .0927734 & .0907231 & 1.02 & 0.307 & -.0851234 & .2706703 \\
\hline _cons | & 13.02959 & $1.78 \mathrm{e}-15$ & $7.3 e+15$ & 0.000 & 13.02959 & 13.02959 \\
\hline
\end{tabular}

Año 2010

Simultaneous quantile regression

Number of obs $=$

2885

bootstrap(20) SES

.50 Pseudo $\mathrm{R} 2=0.0296$

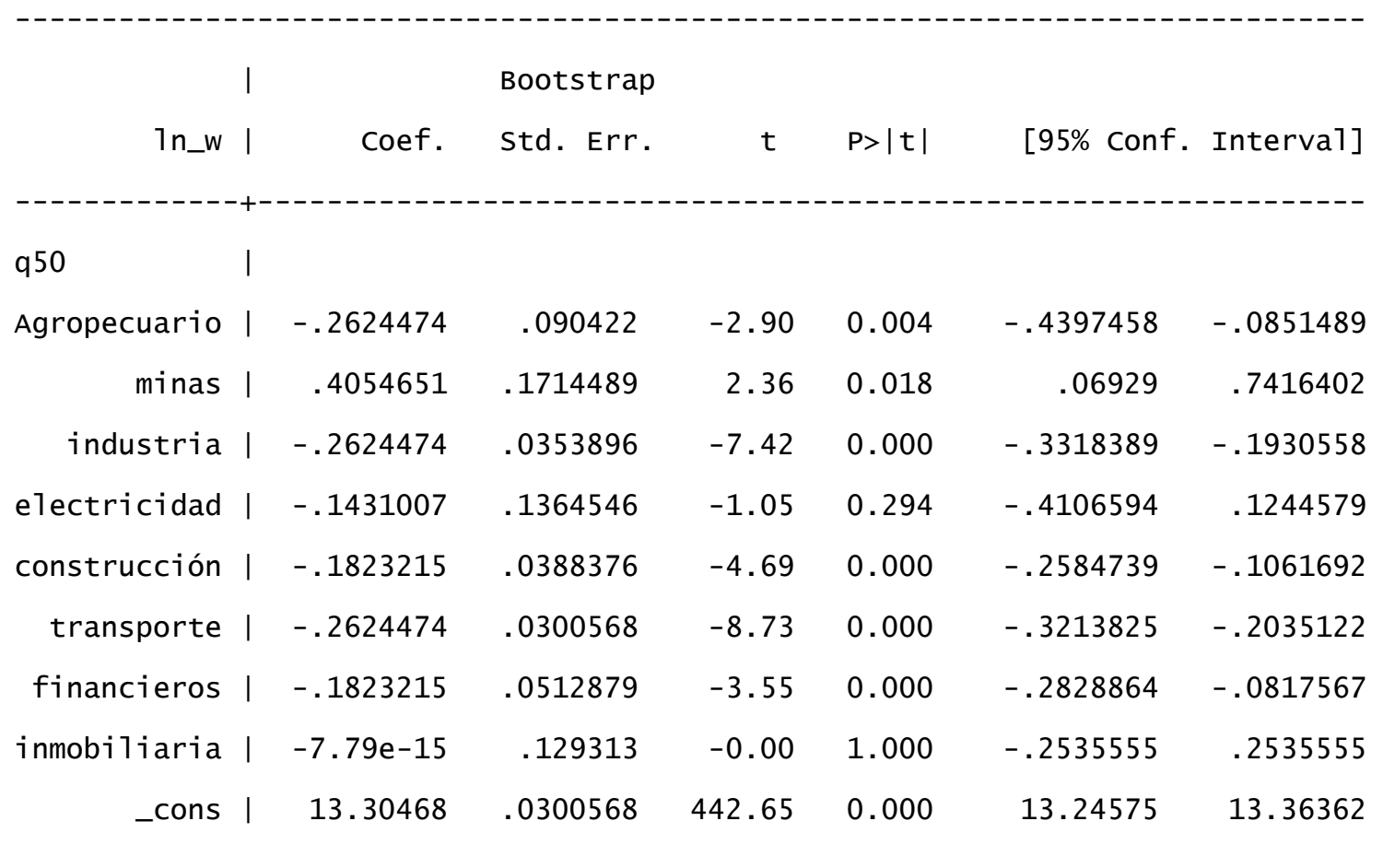

Año 2012

Median regression, bootstrap(20) SES

Raw sum of deviations 1296.926 (about 13.304685)

Min sum of deviations 1224.188
Number of obs $=$

2073

Pseudo R2 $=0.0561$ 


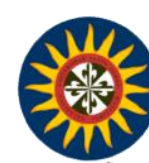

UNIVERSIDAD SANTO TOMAS

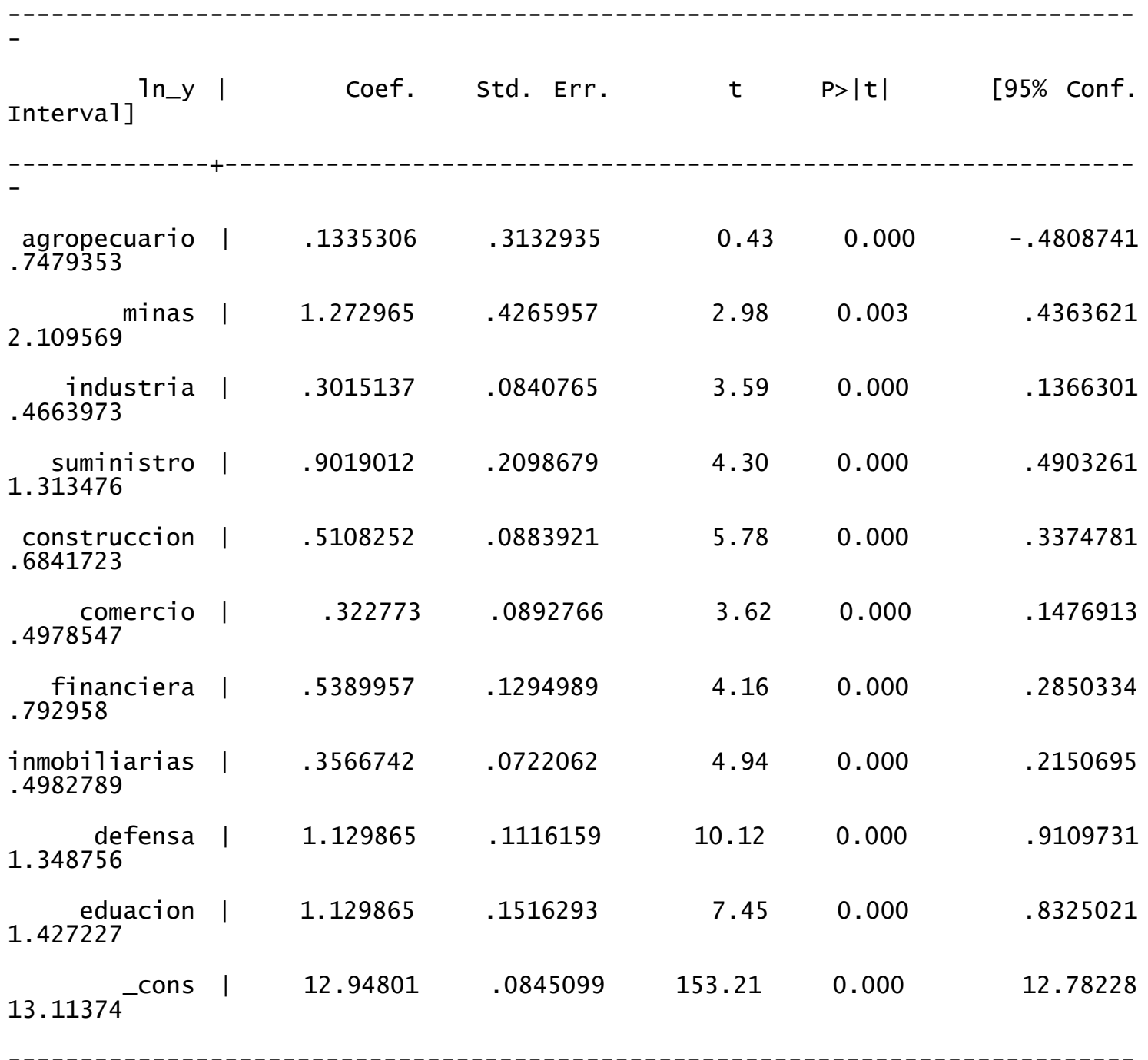

Fuente: DANE, GEIH primer semestre de 2013, cálculos Stata 12 